\title{
Molecular dynamics of cellulose amphiphilicity at the graphene-water interface
}

\author{
Rasha Alqus,,$^{\dagger}$ Stephen J. Eichhorn ${ }^{t^{*}}$ and Richard A. Bryce $e^{\dagger^{*}}$ \\ $†$ Manchester Pharmacy School, University of Manchester, Oxford Road, Manchester, M13 \\ 9PT, UK. \\ \$ Centre for Graphene Science, College of Engineering, Maths \& Physical Sciences, \\ University of Exeter, Physics Building, Stocker Road, Exeter, Devon, EX4 4QL, UK.
}

Cellulose, Graphene, Hydrophobic, Hydrophilic, Amphiphilic, Molecular Dynamics 


\begin{abstract}
Molecular dynamics (MD) simulations have been applied to study the interactions between hydrophobic and hydrophilic faces of ordered cellulose chains and a single layer of graphene in explicit aqueous solvent. The hydrophobic cellulose face is predicted to form a stable complex with graphene. This interface remains solvent-excluded over the course of simulations; the cellulose chains contacting graphene in general preserve intra- and inter-chain hydrogen bonds and a $t g$ orientation of hydroxymethyl groups. Greater flexibility is observed in the more solvent-exposed cellulose chains of the complex. By contrast, the hydrophilic face of cellulose exhibits progressive rearrangement over the course of MD simulations, as it seeks to present its hydrophobic face, with disrupted intra- and interchain hydrogen bonding; residue twisting to form $\mathrm{CH}-\pi$ interactions with graphene; and partial permeation of water. This transition is also accompanied by a more favorable cellulose-graphene adhesion energy as predicted at the PM6-DH2 level of theory. The stability of the cellulose-graphene hydrophobic interface in water exemplifies the amphiphilicity of cellulose and provides insight into favored interactions within graphene-cellulose materials. Furthermore, partial permeation of water between exterior cellulose chains may indicate potential in addressing cellulose recalcitrance.
\end{abstract}




\section{Introduction}

Cellulose is the most used material on the planet and is also the main constituent of plants and trees. Central to its function as a biopolymer is its interaction with water, which primarily arises due to the prevalence of hydroxyl groups along the main backbone of the polymer. ${ }^{1}$ The presence of these hydroxyl groups along the backbone of the polymer chains also allows the formation of hydrogen bonds. These hydrogen bonds occur both along the cellulose chains (intramolecular) and between chains (intermolecular). ${ }^{1}$ Competitive binding with water can occur when forms of cellulose become wet; this leads to, by conventional wisdom, the disruption of hydrogen bonding of the surface chains of cellulose as new hydrogen bonds are formed with the water. ${ }^{1}$ For these very basic reasons, cellulose is often described as a hydrophilic material. Nevertheless, there remains a paradox that cellulose is not dissolvable in neutral $\mathrm{pH}$ water and also is rather recalcitrant to a number of solvents. ${ }^{2}$ One reason suggested for this recalcitrance is the hydrophobic effect that is proposed to occur between chains via the $\mathrm{CH}$ groups, something that is perhaps neglected in considering solvent dissolution. ${ }^{2-3}$ The presence and importance of this hydrophobicity, and the so-called "Lindman effect", has been recently debated ${ }^{3}$ with differing views but nevertheless a call for more research into this effect to take place. Recent molecular modelling and NMR evidence of the binding of caffeine to disaccharides driven by the hydrophobic effect has recently been published. ${ }^{4}$ Hydrophobic effects have also been shown to play a significant role in the formation of crystal-like structures of cellulose ${ }^{5}$ as opposed to a solution state, possibly pointing towards the recalcitrance of the material in common solvents. ${ }^{6}$

The hydrophobic effect itself has been widely studied with respect to the interaction of water with biopolymers. A review by Southall et $a l .{ }^{7}$ has however highlighted some of the difficulties in interpreting the role of entropy and enthalpy on the driving forces for the hydrophobic effect 
with relatively simple systems e.g. oil and water. There have been speculative and seemingly controversial observations of a "sugaring out" effect, with an increased hydrophobic effect between additional components (e.g. amino acid esters) in sugar solutions compared to water. ${ }^{8}$ Increased solubility of otherwise hydrophobic components in water, such as carbon nanotubes, can be achieved through encapsulation using helical polysaccharides such as amylose. ${ }^{9}$ The interaction between amylose and the nonpolar carbon nanotube in aqueous DMSO reflects the amphiphilicity of the polysaccharide. ${ }^{9}$ Other possible evidence of hydrophobic effects include the preferred planar orientation of cellulose chains in nanofibers of bacterial cellulose. ${ }^{10}$

Single layer graphene has emerged, since its discovery by Geim and Noveselov, ${ }^{11}$ as a truly remarkable material. It is known to have high strength, ${ }^{12}$ high conductivity ${ }^{13}$ and is relatively transparent, being only one atom thick. As a result, there is intense interest in utilizing these properties of graphene, including for devices e.g. field effect transistors and also as a transparent conducting material, in combination with other materials, as a potential replacement for indium tin oxide (ITO) in display devices. ${ }^{14}$ Nanocellulose fibers have also emerged as a potential material for a wide range of applications. ${ }^{15}$ The forms of nanocellulose, as nanocrystals (or nanowhiskers) ${ }^{16}$ or nanofibrils from plant material ${ }^{17}$ or bacteria $^{18}$ have typical lateral dimensions $<100 \mathrm{~nm}$ or indeed smaller than the wavelength of visible light. For this reason, they too have been proposed as a potential transparent material for display devices. ${ }^{19}$ Cellulose is non-conductive, and so has been combined with graphene materials as a dielectric material for capacitor devices. ${ }^{20}$ In addition to this, the combination of graphene (and graphene oxide) and cellulose for composite materials has also been proposed. ${ }^{21}$

Given that graphene is thought to have hydrophobic and cellulose amphiphilic properties, it seems apposite to study the effect of water on the interaction between these two materials. In 
order to do this, we report herein a model approach to interrogate the hydrophobic effects between a theoretically constructed cellulose crystal and a single layer of graphene. Little work has been published on modelling the interaction of cellulose and graphene. A recent study ${ }^{22}$ has shown, using a molecular dynamics (MD) simulation, that amorphous cellulose can be reinforced by the presence of graphene and that dispersion of the graphene in the composite aids that process, but this study did not examine the effect of any water. The presence and influence of water on the mechanical properties of a model cellulose crystal has recently been modelled $^{23}$ showing that stiffness increases at a $20 \%$ hydration. In the present study, we show that ordered cellulose chains form a stable complex with graphene in water, most closely interacting along the uniplanar hydrophobic face of the molecule, by seemingly excluding water to the outer edges of the cellulose component, thus demonstrating the amphiphilicity of cellulose. When a hydrophilic face is presented by ordered cellulose chains at the hydrophobic graphene, we find that chains reorient to expose the uniplanar hydrophobic face, again demonstrating the strong interactions between these two materials.

\section{Methods}

\subsection{Molecular dynamics simulations}

A single layer $\mathrm{C}_{2160}$ graphene sheet of area 84.0 x $59.6 \AA^{2}$ was generated by carbon nanotube builder in VMD. ${ }^{24}$ The edges of the sheet were terminated by hydrogen atoms. Two model interfaces between $\mathrm{C}_{2160}$ and cellulose were constructed, selected as the respective model hydrophilic and hydrophobic surfaces of cellulose in order to expose any potential differences in amphiphilic behaviour at the graphene surface. These models were based on the cellulose I $\beta$ crystallographic coordinates reported by Nishiyama et al. ${ }^{25}$ from X-ray fibre diffraction analysis of tunicate cellulose. Firstly, a hydrophobic CH-rich interface, denoted GC100, was

obtained by exposing the 100 face of crystalline cellulose using Cellulose Builder. ${ }^{26}$ The 
cellulose component comprised four layers stacked on the graphene, each containing five decasaccharide chains (Figure 1a). Secondly, a hydrophilic OH-rich interface GC010 was produced along the 010 face, possessing three layers in a 5-4-5 chain arrangement of decasaccharides (Figure 1b). In the absence of graphene, the cellulose systems are labelled C100 and C010 correspondingly. Parameters of zero charged graphene were produced using antechamber assigning the atom type ca from the GAFF force field. ${ }^{27,28}$ Cellulose was modelled using the GLYCAM06h force field. ${ }^{29}$ Scaling factors for 1-4 electrostatic and van der Waals interactions were both taken as unity for cellulose; and the AMBER defaults of 1.2 and 2.0 respectively for graphene. We note that in previous work examining carbohydratearomatic complexes containing $\mathrm{CH}-\pi$ and $\mathrm{OH}-\pi$ interactions, we found that the GLYCAM06 force field yielded interaction energies within $1 \mathrm{kcal} / \mathrm{mol}$ of complete basis set $\operatorname{CCSD}(\mathrm{T})$ values. ${ }^{30}$ For GC100, C100, GC010 and C010, a rectangular box TIP3P water ${ }^{31}$ was added, extending $15 \AA$ A beyond the solute in each dimension.

Molecular dynamics simulations were conducted using the AMBER12 package. ${ }^{27}$ The particle mesh Ewald (PME) method ${ }^{32}$ was used for treatment of long-range electrostatic interactions and a non-bonded cut-off of $10 \AA$ was applied for non-electrostatic interactions. The SHAKE algorithm ${ }^{33}$ was used to constrain covalent bond lengths to hydrogen with a 2 fs time step for dynamics. Simulations were produced in the canonical ensemble, at a temperature of $300 \mathrm{~K}$, controlled with a Langevin thermostat ${ }^{34}$ using a collision frequency of $1 \mathrm{ps}^{-1}$.

The solvated systems were first minimized with restraints on all cellulose and graphene atoms using a force constant of $500 \mathrm{kcal} \mathrm{mol}^{-1} \AA^{-2}$. This was followed by minimization without restraints. The systems were then heated to $300 \mathrm{~K}$ in $50 \mathrm{~K}$ increments over $300 \mathrm{ps,} \mathrm{with}$ restraints on cellulose and graphene using a force constant of $500 \mathrm{kcal} \mathrm{mol}^{-1} \AA^{-2}$. Equilibrations 
began with $1 \mathrm{~ns}$ of NVT dynamics with gradual reduction of restraints on cellulose (to zero) and graphene atoms (to $10 \mathrm{kcal} \mathrm{mol}^{-1} \AA^{-2}$ ), followed by $1 \mathrm{~ns}$ each of NVT, NPT and NVT dynamics consecutively. Subsequently NVT simulations of $300 \mathrm{~ns}$ were acquired. Additional 100 ns simulations for GC100 and GC010 were obtained by applying different input pseudorandom values for Langevin dynamics after the initial equilibration, as well as $100 \mathrm{~ns}$ simulations for each of $\mathbf{C 1 0 0}$ and $\mathbf{C 0 1 0}$.

\subsection{Analysis}

Root mean square deviation (RMSD) in position of cellulose atoms over the MD simulations was computed, excluding the C6 hydroxymethyl group and C2 and C3 hydroxyl groups. Glycosidic torsion angles $\varphi$ and $\psi$ between glucosyl rings $i$ and $i-1$ were defined by atoms $\mathrm{O} 5(i)-\mathrm{C} 1(i)-\mathrm{O} 4(i-1)-\mathrm{C} 4(i-1) \quad$ and $\quad \mathrm{C} 1(i)-\mathrm{O} 4(i-1)-\mathrm{C} 4(i-1)-\mathrm{C} 5(i-1), \quad$ respectively. $\quad$ The hydroxymethyl group orientation $\omega$ was defined by $\mathrm{O} 5(i)-\mathrm{C} 5(i)-\mathrm{C} 6(i)-\mathrm{O} 6(i)$ and $\mathrm{C} 4(i)-\mathrm{C} 5(i)-\mathrm{C} 6(i)-\mathrm{O} 6(i)$ respectively.

Intramolecular and intermolecular hydrogen bonds, for donor atoms D-H and acceptor atom A, were defined as having a $\mathrm{H} \cdots$ A distance $<3.5 \AA$ and $\mathrm{D}-\mathrm{H} \cdots$ A angle $>120^{\circ} .{ }^{35}$ Using features employed by Mohan et al., ${ }^{36} \mathrm{CH}-\pi$ and $\mathrm{OH}-\pi$ interactions were deemed present if (i) the distance between the hydrogen atom of the $\mathrm{CH}$ or $\mathrm{OH}$ group and the centroid of the six-carbon $\pi$ system lies in range of $2.6-3.0 \AA$, (ii) the angle between the $\mathrm{C}-\mathrm{H}$ or $\mathrm{O}-\mathrm{H}$ bond and the centroid of the $\pi$ system is $\geq 120^{\circ}$, and (iii) the distance between the heavy atom of the $\mathrm{CH}$ or OH group and the centroid of the $\pi$ system is $\leq 4.5 \AA$. All geometric analyses were performed using the ptraj and cpptraj modules ${ }^{37}$ of AMBER. 
The adhesion energy for graphene with cellulose $\left(E_{\text {inter }}\right)$ was computed in vacuo using single point energy calculations at the PM6-DH2 level of theory ${ }^{38}$ based on geometries obtained from the simulations. This semi-empirical MO level of theory, corrected for dispersive interactions, has been employed for interactions of small molecules, including with graphene, ${ }^{39}$ for example reproducing well the experimental adsorption energy of aromatic hydrocarbons on graphene. ${ }^{40}$ Adhesion energies in solution were obtained using PM6-DH2 in conjunction with the COSMO implicit solvent model ${ }^{41}$ using a dielectric constant of 78.4. Whilst COSMO has its limitations as an implicit solvent model $^{42}$, recent work has shown PM6-DH2 calculations in COSMO solvent to give generally good agreement with potential of mean force calculations of adsorption energies in explicit aqueous solvent for compounds on graphene. ${ }^{28}$ The adhesion energy in gas-phase and solution was computed as an average of interaction energies computed for structures taken at $20 \mathrm{~ns}$ intervals over the last $100 \mathrm{~ns}$ of the trajectory. All quantum chemical calculations were performed with MOPAC version $14.128 \mathrm{~L}^{42}$

\section{Results}

We consider the structure, stability and interactions of a hydrophobic and hydrophilic cellulose surface with a $\mathrm{C}_{2160}$ graphene sheet. The pre-formed graphene-cellulose complexes, labelled GC100 (Figure 1a) and GC010 (Figure 1b) respectively, are modelled in explicit aqueous solvent using $300 \mathrm{~ns}$ molecular dynamics simulations at $300 \mathrm{~K}$. We also compare with additional 100 ns simulations of GC100 and GC010, and 100 ns simulations of the two solvated cellulose models in the absence of graphene (denoted $\mathbf{C 1 0 0}$ and $\mathbf{C 0 1 0}$ respectively).

\subsection{Hydrophobic 100 interface}

The hydrophobic interfacial model GC100 largely retained its initial structural configuration over the course of the $300 \mathrm{~ns}$ MD simulation. As illustrated from the time series of snapshots 
for this simulation (Figure 2a), the layered structure of cellulose remains broadly intact on the graphene surface, although some significant variations in glucosyl residues of the most solvent exposed layer (layer 4) is observed. By the end of a $4 \mathrm{~ns}$ equilibration, the cellulose has optimised its complementarity with the graphene (Figure 2a). The graphene itself displays an undulating surface that oscillates to a limited extent over the simulation, despite the presence of the mild tethering restraints; indeed, the flexibility of single layer graphene sheets has been computationally studied previously. ${ }^{43}$ Layer 1 , the layer immediately adjacent to the $\mathrm{C}_{2160}$ surface, appears particularly stable around its initial orientation (Figure 2b); this is also found for the shorter $100 \mathrm{~ns}$ simulation (Figure S1). Layer 1 has an all atom RMSD of $0.3 \AA$ on average across its cellulose chains (Table 1, Figure 3a). This RMSD value is small with respect to layer 4, the outermost layer in the complex, which has an average of $0.5 \AA$ (Table 1). We contrast this observed stability of $\mathbf{G C 1 0 0}$ with the simulation of $\mathbf{C 1 0 0}$, namely the cellulose component in the absence of graphene. For $\mathbf{C 1 0 0}$, considerable twisting of the cellulose is observed as the system seeks to minimise the water exposure of its hydrophobic 100 face (Figure S1a). For example, the difference between the curvature of layer 1 of $\mathbf{C 1 0 0}$ and the planarity of GC100 is very apparent (Figure S1b). The higher flexibility of $\mathbf{C 1 0 0}$ compared to GC100 is reflected by a respective RMSD of $0.6 \AA$ compared to $0.3 \AA$ for layer 1 (Table S1).

The stability of the graphene-cellulose interface in the GC100 simulation is reflected by a relatively constant interfacial surface area, which is $5029 \AA^{2}$ after equilibration and $5085 \AA^{2}$ at the end of the simulation. Similarly, there is a conservation of the initially formed $\mathrm{CH}-\pi$ interactions across the interface; according to our definition in Methods, these interactions number $\sim 190$ (Figure 4). There is also an almost complete absence of $\mathrm{OH}-\pi$ interactions sampled during the MD (Figure 4). Therefore, using the three geometric criteria defined in Methods, we count on average just under four $\mathrm{CH}-\pi$ interactions per saccharide residue at the 
interface; three of the interactions correspond to alternately (i) the glucosyl ring axial $\mathrm{C} 1, \mathrm{C} 3$ and $\mathrm{C} 5$ protons or (ii) $\mathrm{C} 2$ and $\mathrm{C} 4$ axial protons and along with one of the two $\mathrm{C} 6$ methylene $\mathrm{H}$ atoms (due to the 2 -fold screw axis of the cellulose chains). From the simulations, the remaining fractional interaction appears to arise from transient fluctuations in hydroxyl and hydroxymethyl groups, which are of greater prominence for solvent exposed residues.

At the PM6-DH2 level of theory, the total gas phase adhesion energy of the GC100 graphenecellulose complex is $-784 \mathrm{kcal} \mathrm{mol}^{-1}$; note that this energy is an average of adhesion energies computed for snapshots taken at $20 \mathrm{~ns}$ intervals over the last $100 \mathrm{~ns}$ of the trajectory (Figure 5a). Making the assumption that this total interaction energy solely arises from $\mathrm{CH}-\pi$ interactions, this gives a value of $4.0 \mathrm{kcal} \mathrm{mol}^{-1}$ per $\mathrm{CH}-\pi$ interaction; this is similar to the estimate of $4.1 \mathrm{kcal} \mathrm{mol}^{-1}$ from the $100 \mathrm{~ns} \mathbf{G C 1 0 0}$ simulation (Figures S2a and S3a). These values compare to a computed adsorption energy of $3.1 \mathrm{kcal} \mathrm{mol}^{-1}$ for methane on a $\mathrm{C}_{54}$ graphene core at the PM6-DH2 level ${ }^{40}$ beyond the simplicity of the above assumption, the slightly higher estimate we find could reflect an enhanced effect due to multiple $\mathrm{CH}-\pi$ interactions, as has been suggested elsewhere. ${ }^{44}$ On solvation, modelled by COSMO implicit solvent, the total PM6-DH2 interaction energy drops to an average of $-549 \mathrm{kcal} \mathrm{mol}^{-1}$ over the last $100 \mathrm{~ns}$ (Figure 5b), leading to an estimate of $-2.9 \mathrm{kcal} \mathrm{mol}^{-1}$ per $\mathrm{CH}-\pi$ interaction. As mentioned above, there is an absence of $\mathrm{OH}-\pi$ interactions sampled during the simulations of GC100 (Figure 4), further underlining the hydrophobic nature of this interface. Indeed, inspection of the water number density indicates total exclusion of water at the interface over the course of the simulation (Figures 6 and S4); this can be most clearly seen when only the solvent is visualized (Figure 6). 
The stability at this hydrophobic cellulose-graphene interface is also reflected by the internal geometry of the cellulose chains in layer 1: glycosidic linkages between two D-glucopyranose units in this layer remain close to their initial $\mathrm{I}_{\beta}$ orientations $\varphi$ and $\psi$ of $-95.6^{\circ}$ and $-145.8^{\circ}($ e.g . chain 5 in Figure 7a). Experimentally, the average value of $\varphi$ and $\psi$ in cellulose fibril are $-88.7^{\circ}$ and $-147.1^{\circ}$ for center chains; and $-98.5^{\circ}$ and $-142.3^{\circ}$ origin chains, respectively. ${ }^{24}$ The orientation $\omega$ of the C6 hydroxymethyl groups plays an important role in the stability of cellulose due its control of the type of hydrogen bond formed. ${ }^{45}$ During the MD simulations of GC100, these groups remain principally although not exclusively in their initial crystallographic $\operatorname{tg}$ orientations (Figure 8a). The $\operatorname{tg}$ orientation allows the formation of an intramolecular hydrogen bond in the same chain and intermolecular hydrogen bond with adjacent chains. ${ }^{46}$ This orientation has been observed to persist for interior chains in simulations of hydrated cellulose fibril using the GLYCAM06 and CHARMM35 force fields, ${ }^{47}$ although to a much greater extent in the former; the degree of stabilisation of $t g$ conformer by GLYCAM06 has been questioned. ${ }^{48}$ In our 300 ns simulation of GC100, we find the outer chains that contact water variously sample predominately $g t, g g$ or $t g$ conformations (Figure 8a). Similar results for interior and exterior chains are obtained for the $100 \mathrm{~ns}$ simulation of GC100 (Figure S5a).

The structure of cellulose $\mathrm{I}_{\beta}$ contains a network of intra- and interchain hydrogen bonds in the A scheme (Figure S6). ${ }^{46}$ In our simulations of GC100, we see good preservation of the $\mathrm{I}_{\beta}$ intrachain $\mathrm{O} 3-\mathrm{H} \cdots \mathrm{O} 5$ and $\mathrm{O} 2-\mathrm{H} \cdots \mathrm{O} 6$ hydrogen bonds within this layer, with an average value of 8 and 6 bonds per decasaccharide chain respectively during the $300 \mathrm{~ns}$ MD simulation (Figure 9a). Similarly, the $\mathrm{I}_{\beta}$ interchain $\mathrm{O} 6-\mathrm{H} \cdots \mathrm{O} 2$ and $\mathrm{O} 6-\mathrm{H} \cdots \mathrm{O} 3$ hydrogen bonds persist with values of 5 and 8 interactions respectively (Figure 9a). A similar pattern is observed from the $100 \mathrm{~ns}$ simulation (Figure S7). 
As expected for the outer and most solvent exposed layer of cellulose, the average chain RMSD of layer 4 in $\mathbf{G C 1 0 0}$ is $0.5 \pm 0.4 \AA$, considerably higher than that of layers 1-3 (Table 1, Figure 3a); for example, layer 1 has an average chain RMSD of $0.3 \pm 0.1 \AA$ (Table 1 ). The higher disorder of this more solvent exposed layer of chains in GC100 is also evident from inspection of MD snapshots, where individual saccharide residues periodically project out into solution (Figure 2a) through twisting (Figure 2c). Correspondingly, there is somewhat greater exploration of $\varphi \psi$ orientations (eg. compare Figure 7a with S8), and hydroxymethyl orientations $g g / g t$ (Figures 8a and S5a) as observed for solvent exposed exterior chains in simulations of a solvated cellulose fibril using GLYCAM06. ${ }^{48}$ Competition with solvent leads to decreased intra-chain hydrogen bonding (from 8 to 4 interactions per decasaccharide chain for $\mathrm{O} 2-\mathrm{H} \cdots \mathrm{O} 6$, Figure 9a). However, while the inter-chain $\mathrm{O} 6-\mathrm{H} \cdots \mathrm{O} 3$ interaction is similarly

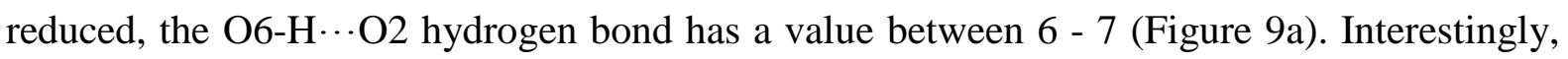
when we simulate the cellulose of $\mathbf{G C 1 0 0}$ for 100 ns in water but in the absence of graphene, we find that the intra-chain $\mathrm{O} 2-\mathrm{H} \cdots \mathrm{O} 6$ hydrogen bonds in the exposed layers 1 and 4 were lost completely by the end of the trajectory (data not shown). For GC100, despite some depletion in its internal hydrogen bonding for layer 4, however, the overall structure of the cellulosegraphene complex is remarkably well maintained during the simulation, indicating strong complementarity of graphene with the 100 surface of cellulose.

\subsection{Hydrophilic 010 interface}

In contrast to the stable hydrophobic GC100 system, major rearrangement of the cellulose chains with respect to graphene was observed along its hydrophilic 010 face in the GC010 simulations. MD snapshots over the $300 \mathrm{~ns}$ reveal progressive rotation of glucosyl ring orientations such that their $\mathrm{CH}$ rather than $\mathrm{OH}$ groups orient towards the phenyl rings of graphene (Figure 10a-p). This feature is evident from 5 ns onwards (yellow residues, Figure 
10e) and first appears from the reducing end of the cellulose chains in layer 1 (Figure 10e) but shortly after from the non-reducing ends (Figure 10f). By $14 \mathrm{~ns}$, four out of the five chains contain at least one twisted residue (Figure 10g). Interestingly, at 20 ns in the MD simulation over the space of $100 \mathrm{ps}$, there is a fairly abrupt transition such that $42 \%$ of the residues in layer 1 are rotated (Figures $10 \mathrm{i}$ and $10 \mathrm{j}$ ). At this point in the $300 \mathrm{~ns}$ simulation, chain 1 from layer 2 is able to descend such that its $\mathrm{OH}$ groups can form direct interactions with the graphene surface (orange chain, Figure 10j). By 100 ns, $48 \%$ of residues in the six chains had twisted (Figure 10m); by $184 \mathrm{~ns}$, the descended layer 2 chain starts to twist to present $\mathrm{CH}$ groups to graphene (Figure 10n); $58 \%$ of residues in these chains had twisted by $300 \mathrm{~ns}$ (Figure 10o). This rearrangement is mirrored by the change in the graphene-cellulose interfacial surface area, from $2149 \AA^{2}$ after equilibration to $4294 \AA^{2}$ by the end of the simulation. A similar degree of chain reorientation was also observed for the second MD simulation of CG010, although more gradually and with no chains from layer 2 entering into layer 1 on the 100 ns timescale (Figure S9). Interestingly, for the graphene-free simulation of the 010 model in water, C010, we find significant overall twisting in the cellulose assemblage (Figure SXa), as was observed for C100 (Figure S1a). Once again, the difference between the curvature of layer 1 of the graphene-free system and planarity of the graphene-bound system is marked (Figure S1b). However, due to the reorganisation of the 010 face to form a 100 face at the graphene surface, a higher RMSD is in fact observed for GC010 (1.1 $⿱$, Table S1) compared to C010 (0.9 $)$ ). We also note that while rotation of specific residues does occur within the cellulose chains of $\mathbf{C 0 1 0}$, this appears random and reversible in comparison to the systematic formation of a 100 face at the graphene surface in the GC010 simulations (Figure S12X).

We can directly chart the progressive replacement of layer $1 \mathrm{OH}-\pi$ interactions with a greater number of $\mathrm{CH}-\pi$ interactions: initially the GC010 model contains $30 \mathrm{OH}-\pi$ interactions (Figure 
4), with a corresponding interaction energy in vacuo of $-92 \mathrm{kcal} \mathrm{mol}^{-1}$ (Figure 5a). This equates to $3.0 \mathrm{kcal} \mathrm{mol}{ }^{-1}$ per $\mathrm{OH}-\pi$ interaction. This value is slightly less than the estimate of $-4.2 \mathrm{kcal}$ mol $^{-1}$ at the PM6-DH2 level for the interaction energy of water with a $\mathrm{C}_{54}$ graphene core. ${ }^{39}$ However, there is a drop in the number of $\mathrm{OH}-\pi$ interactions, from an initial value of 30 to an average value of $\sim 9$ interactions by $100 \mathrm{~ns}$, involving, in part, $\mathrm{OH}$ groups from chain 1 from layer 2. There is a growth in the number of $\mathrm{CH}-\pi$ interactions during $4 \mathrm{~ns}$ from zero to 31 , and subsequently up to $\sim 85$ by $300 \mathrm{~ns}$ (Figure 4). Correspondingly, the adsorption energy increases significantly to an average over the last $100 \mathrm{~ns}$ of $-520.1 \mathrm{kcal} \mathrm{mol}^{-1}$ (Figure 5a). In implicit solvent, the computed adsorption energy is $-296.0 \mathrm{kcal} \mathrm{mol}^{-1}$ over the last $100 \mathrm{~ns}$ (Figure $5 \mathrm{~b}$ ). This loss of $43 \%$ of the interaction energy in the GC010 complex on solvation compares with a reduction of only $30 \%$ for $\mathbf{G C 1 0 0}$, indicating the greater ease of desolvating the hydrophobic 100 face of cellulose.

Reflecting the progressive rearrangement of GC010, the all atom RMSD for the cellulose chains of GC010 exhibit stepwise shifts over time (Figure 3b), for example, at $80 \mathrm{~ns}$ for chain 1 of layer 1; 130 ns for chain 1 of layer 2; and 100 ns for chain 5 of layer 3 . The chain RMSD values are also higher than those of hydrophobic GC100, rising to values of $2 \AA$, or, for chain 1 of the outermost layer 3, up to $3 \AA$ (Figure 3b). For chain RMSD, layer 1 of GC010, where most geometry changes arise, had an average RMSD of $1.1 \AA$ (Table 1 ), compared to $0.3 \AA$ found for layer 1 of $\mathbf{G C 1 0 0}$. In layer 2 of GC010, chain 1 had the highest RMSD, of $0.9 \AA$, compared to the rest of the chains in the layer; this is the chain that migrated from layer 2 to layer 1. Similarly, chain 1 of layer 3 moves into layer 2, with an associated RMSD value of 1.9 Å (Table 1). 
In GC010, the glycosidic torsions exhibited considerable fluctuation. For example, we consider chain 5 of layer 1: here, the glycosidic linkages between the first two D-glucopyranose units (numbered 1 and 2) deviate transiently from their initial $\mathrm{I}_{\beta}$ orientations of $-95.6^{\circ}$ and $-145.8^{\circ}$ in the first $50 \mathrm{~ns}$ (Figure $7 \mathrm{~b}$ ), increasing to $-48.2^{\circ}$ and $-90.6^{\circ}$ respectively. These transitions reflect the relaxation of the chain as it seeks to maximise hydrophobic interactions with the graphene surface (Figure 10).

In contrast to the dry graphene-cellulose interface of GC100, the grooved GC010 interface is penetrated with water (Figures 6 and S4). The propensity of the 010 plane for localised water interactions has previously been observed from MD simulation of cellulose in water ${ }^{46}$ Interestingly, as glucosyl residues rotate to form hydrophobic contacts with graphene, water is gradually expelled at the graphene-cellulose interface (Figure 6a-f), with a reduction of $\sim 30 \%$. As the chains rearrange, however, bulk water molecules appear able to insert between the more exterior chains (Figure 6c-e). This can be observed, for example, between chain 1 of layer 1 and adjacent cellulose chains from layers 1,2 and 3 (Figure 11). The presence of water at the interface also appears linked to hydroxymethyl conformational changes to favor $g t / g g$ in layer 1 (Figure 8b). The inner chains that are not surrounded by water maintain a mainly $t g$ conformation; otherwise, the remaining chains change orientation to $g t / g g$ with a minor amount of $t g$, in agreement with a NMR study of the crystalline cellulose-water interface. ${ }^{47}$ Similar cellulose $\mathrm{CH}_{2} \mathrm{OH}$ preferences can be observed in the 100 ns GC010 simulation (Figure S5b).

In regard to hydrogen bonding in $\mathbf{G C 0 1 0}$, the number of intrachain $\mathrm{O} 3-\mathrm{H} \cdots \mathrm{O} 5$ hydrogen bonds in layer 1, as defined according to the initial crystallographic hydrogen bond network, is stable at a value of around 8 (Figures $10 \mathrm{~b}$ and S7b). This was also the case for GC100 (Figure 10a). However, in this layer, the initial intrachain hydrogen bond, $\mathrm{O} 2-\mathrm{H} \cdots \mathrm{O} 6$, is gradually lost over 
the course of the simulation of GC010, decreasing from 8 to 2 per decasaccharide chain on average (Figures $10 \mathrm{~b}$ and S7b). This pattern of initial loss between hydrogen bond partners is similarly observed for interchain hydrogen bonds for layers 1, 2 and 3 (layers 1 and 3 shown in Figure 10b). However, as the chains reorient, they partner with different chains, in the A hydrogen bonding pattern, as can be seen from the gradual growth in new interchain $\mathrm{O} 6-\mathrm{H} \cdots \mathrm{O} 3$ and O6-H $\cdots \mathrm{O} 2$ hydrogen bonds (Figures 12 and S10). The transient severing of interchain hydrogen bonds and changes in glycosidic and hydroxymethyl torsion values are indicative of the rearrangement in chain orientation as the cellulose adapts to the graphene surface. Considering chain 5 in layers 1 and 3 as an example, the initial 010 orientation of the cellulose has the 6-OH groups of this chain facing in the direction of the graphene surface (Figure 13). However, the hydrogen bonds between terminal residues at one end of these chains are broken by $14 \mathrm{~ns}$ in the $300 \mathrm{~ns}$ simulation and $7 \mathrm{~ns}$ in the $100 \mathrm{~ns}$ simulation (Figure 13); shortly afterwards, the interchain contacts at both ends of the chain sever, with the layer 1 chain rotating to form $\mathrm{CH}-\pi$ interactions with graphene. This is observed the snapshots of $\mathbf{G C 1 0 0}$ at $104 \mathrm{~ns}$ and $50 \mathrm{~ns}$ for the $300 \mathrm{~ns}$ and $100 \mathrm{~ns}$ simulations respectively (Figure 13). These contacts progressively extend from both ends of the chain until $\mathrm{CH}$ contacts are made by almost all residues by the end of the two simulations (Figure 12). Additionally, we observe the layer 3 chain beginning to rotate into a similar orientation as layer 1 (data not shown).

\section{Discussion}

In this work, we have evaluated the interactions between model hydrophobic and hydrophilic faces of ordered cellulose chains and a single layer of graphene, in the presence of water. We find from molecular dynamics simulations that the hydrophobic 100 surface of cellulose is stabilised by the presence of graphene. This adhesion appears to occur principally through interactions between its glucopyranose $\mathrm{CH}$ groups and the graphene $\pi$ electron system, which 
appear competitive in affinity with cohesive inter-cellulose energies. Our simulations indicate that cellulose is able to present a high density of these $\mathrm{CH}$ groups, achieving 3 - 4 interactions per residue. Due to solvation of this complex, the water-exposed cellulose chains show greater flexibility than interior and graphene-facing chains but nevertheless the assembly retains its integrity.

The cellulose 010 face presents $\mathrm{OH}$ groups towards the graphene surface. The hydroxyl groups are also capable of good affinity with $\pi$ electron systems, and have been estimated from high level ab initio QM calculations as between -3 to $-5 \mathrm{kcal} \mathrm{mol}^{-1}$ in affinity. ${ }^{43}$ In this system, however, a gradual large scale reorientation of cellulose occurs, to form the $\mathrm{CH}-\pi$ interactions as found for the 100 face. Whilst the comparable graphene-free simulation shows overall twisting of the cellulose, there is not the systematic formation of the hydrophobic surface observed in the presence of graphene. Indeed, the mechanism of this rearrangement at the graphene surface appears to involve temporary interruption of chain-chain hydrogen bonds beginning at terminal residues, facilitated by the aqueous environment. This severing of interchain interactions allows for twisting around the glycosidic linkages such that the glucopyranose $\mathrm{CH}$ rather than $\mathrm{OH}$ groups present towards the graphene surface. Subsequent reformation of chain-chain interactions occurs, along with progressive expulsion of waters found at the interface.

The results of this work clearly point to the amphiphilic and adaptable nature of cellulose when challenged with this biphasic polar-nonpolar environment. Indeed, such carbohydrate- $\pi$ interactions have been found to play a significant role for example in the selective interaction of carbohydrate-binding module $(\mathrm{CBM})$ with hydrophobic cellulose surfaces via a triad of tyrosine residues. ${ }^{49}$ Similarly, $\mathrm{CH}-\pi$ interactions are observed in recognition of sugar substrates 
more generally: ${ }^{50}$ for example, this is found in the recognition of the lactosyl motif by stacking between tryptophan and tyrosine residues in the acceptor binding site of Trypanosoma cruzi trans-sialidase. ${ }^{51}$ Conversely, when confronted with the nonpolar graphene surface, we witness the rearrangement of the 010 cellulose model in our simulations to orient more $\mathrm{CH}$ groups at graphene. Indeed, graphene is known to interact strongly with hydrophobic components of other biomolecules, potentially altering their conformation and disrupting their biological activity. For example, direct adhesion of concanavalin A to single-layer graphene led to a loss in its function, suggesting structural rearrangement in this normally very stable $\beta$-sandwich protein. ${ }^{52}$ These $\mathrm{CH}-\pi$ interactions are likely to dominate within amorphous cellulose-graphene composites, which form flexible, conductive and mechanically strong materials.

The stability of the hydrophobic 100 face of cellulose with graphene in water exemplifies the amphiphilicity of cellulose and provides insight into favored interactions within graphenecellulose nanocomposites. For the simulations of the 010 face at graphene, we observe a reorganisation of cellulose chains that appears systematic and distinct from graphene-free simulation. Interestingly, this transition includes disruption of interchain hydrogen bonds. This weakening of interchain hydrogen bonds, coupled to a degree of water permeation between the more solvent exposed cellulose chains (GC010 in Figure 11), could be of potential benefit in allowing penetration into cellulose of solvents besides water; thus the presence of graphene could promote solvolysis of the glycosidic linkages in this notoriously recalcitrant material. Nevertheless, we note that in this exploration of amphiphilicity, these are model systems involve relatively small cellulose cross-sections designed to present distinctly hydrophobic and hydrophilic faces at the graphene surface. Future work is required to examine the atomistic behaviour of cellulose models of greater extent at graphene surfaces as well as cellulose faces of intermediate hydrophobicity, specifically the 110 and $1 \overline{1} 0$ surfaces. However, the predicted 
competition between graphene and cellulose for interaction with $\mathrm{OH}$ and $\mathrm{CH}$ groups is intriguing and likely to be reflected at the very least in boundary regions where water, graphene and cellulose intersect.

\section{Conclusions}

It has been shown that the graphene-cellulose interface is an interesting one from dual perspectives. Firstly, it offers a route to assembly in water of a potentially strong and stiff composite; and secondly, it allows, in a modelling environment, predictions about the amphiphilic nature of cellulose. Molecular dynamics simulations in explicit aqueous solvent suggest a stable hydrophobic interface can be formed between ordered cellulose chains and a graphene surface, formed predominately by $\mathrm{CH}-\pi$ interactions. However, simulations reveal significant rearrangement of a hydrophilic cellulose surface in the presence of graphene and water, such that $\mathrm{CH}-\pi$ interactions with graphene are maximised and occluded from a polar solvent. In the process of this rearrangement, water is predicted to partially penetrate the cellulose structure. This behaviour serves to highlight the crucial amphiphilic nature of carbohydrate polymers in general, and in practical terms may offer opportunities in tackling cellulose recalcitrance.

\section{Supporting Information}

Additional details of the analysis of replicate and reference molecular dynamics simulations are provided in Supporting Information. This material is available free of charge via the Internet at http://pubs.acs.org. 


\section{References}

1. Klemm, D.; Philipp, B.; Heinze, T.; Heinze, U.; Wagenknecht, W., Comprehensive Cellulose Chemistry: General Principles and Analytical Methods. Wiley-VCH: Weinheim: 1998.

2. Lindman, B.; Karlstrom, G.; Stigsson, L. J. Mol. Liq. 2010, 156, 76-81.

3. Glasser, W.; Atalla, R.; Blackwell, J.; Malcolm Brown, R., Jr.; Burchard, W.; French, A.; Klemm, D.; Nishiyama, Y. Cellulose 2012, 19, 589-598.

4. Tavagnacco, L.; Engstrom, O.; Schnupf, U.; Saboungi, M.-L.; Himmel, M.; Widmalm, G.; Cesaro, A.; Brady, J. W. J. Phys. Chem. B 2012, 116, 11701-11711.

5. French, A. D.; Concha, M.; Dowd, M. K.; Stevens, E. D., Electron (charge) density studies of cellulose models. Cellulose 2014, 21 (2), 1051-1063.

6. Bergenstrahle, M.; Wohlert, J.; Himmel, M. E.; Brady, J. W. Carbohydr. Res. 2010, 345, 2060-2066.

7. Southall, N. T.; Dill, K. A.; Haymet, A. D. J. J. Phys. Chem. B 2002, 106, 521-533.

8. Lakshmi, T. S.; Nandi, P. K. J. Chromatogr. A 1976, 116, 177-179

9. Kim, O.-K.; Je, J.; Baldwin, J. W.; Kooi, S.; Pehrsson, P. E.; Buckley, L. J. J. Am. Chem. Soc. 2003, 125, 4426-4427.

10. Bohn, A.; Fink, H.-P.; Ganster, J.; Pinnow, M. Macromol. Chem. Phys. 2000, 201, 1913-1921.

11. Novoselov, K. S.; Geim, A. K.; Morozov, S. V.; Jiang, D.; Zhang, Y.; Dubonos, S. V.; Grigorieva, I. V.; Firsov, A. A. Science 2004, 306, 666-669.

12. Rasool, H. I.; Ophus, C.; Klug, W. S.; Zettl, A.; Gimzewski, J. K. Nat Commun 2013, 4, 2811.

13. Novoselov, K. S.; Geim, A. K.; Morozov, S. V.; Jiang, D.; Katsnelson, M. I.;

Grigorieva, I. V.; Dubonos, S. V.; Firsov, A. A. Nature 2005, 438, 197-200. 
14. Khrapach, I.; Withers, F.; Bointon, T. H.; Polyushkin, D. K.; Barnes, W. L.; Russo, S.; Craciun, M. F. Adv. Mater. 2012, 24, 2844-2849.

15. (a) Klemm, D.; Kramer, F.; Moritz, S.; Lindström, T.; Ankerfors, M.; Gray, D.; Dorris, A. Angew. Chem., Int. Ed. 2011, 50, 5438-5466. (b) Eichhorn, S. J.; Dufresne, A.; Aranguren, M.; Marcovich, N. E.; Capadona, J. R.; Rowan, S. J.; Weder, C.; Thielemans, W.; Roman, M.; Renneckar, S.; Gindl, W.; Veigel, S.; Keckes, J.; Yano, H.; Abe, K.; Nogi, M.; Nakagaito, A. N.; Mangalam, A.; Simonsen, J.; Benight, A. S.; Bismarck, A.; Berglund, L. A.; Peijs, T. J. Mater. Sci. 2010, 45, 1-33.

16. Eichhorn, S. J. Soft Matter 2011, 7, 303-315.

17. Siro, I.; Plackett, D. Cellulose 2010, 17, 459-494.

18. Klemm, D.; Schumann, D.; Udhardt, U.; Marsch, S. Prog. Polym. Sci. 2001, 26, 15611603.

19. (a) Nogi, M.; Yano, H. Adv. Mater. 2008, 20, 1849-1852. (b) Nogi, M.; Iwamoto, S.;

Nakagaito, A. N.; Yano, H. Adv. Mater. 2009, 21, 1595-1598.

20. Weng, Z.; Su, Y.; Wang, D.-W.; Li, F.; Du, J.; Cheng, H.-M. Adv. Energy Mater. 2011, $1,917-922$.

21. (a) Tian, M.; Qu, L.; Zhang, X.; Zhang, K.; Zhu, S.; Guo, X.; Han, G.; Tang, X.; Sun, Y. Carbohydr. Polym. 2014, 111, 456-462. (b) Valentini, L.; Cardinali, M.; Fortunati, E.; Kenny, J. M. Appl. Phys. Lett 2014, 105, 153111. (c) Rahman, R.; Haque, A. Procedia Eng. 2013, 56, 789-794. (d) Zhang, J.; Cao, Y.; Feng, J.; Wu, P. J. Phys. Chem. C 2012, 116, 8063-8068. (e) Kabiri, R.; Namazi, H. Cellulose 2014, 21, 3527-3539.

22. Rahman, R.; Foster, J. T.; Haque, A. J. Phys. Chem. A 2013, 117, 5344-5353.

23. Petridis, L.; O'Neill, H. M.; Johnsen, M.; Fan, B.; Schulz, R.; Mamontov, E.; Maranas, J.; Langan, P.; Smith, J. C. Biomacromolecules 2014, 15, 4152-4159.

24. Humphrey, W.; Dalke, A.; Schulten, K. J. Molec. Graphics Model. 1996, 14, 33-38. 
25. Nishiyama, Y.; Langan, P.; Chanzy, H. J. Am. Chem. Soc. 2002, 124, 9074-9082.

26. Gomes, T. C. F.; Skaf, M. S. J. Comput. Chem. 2012, 33, 1338-1346.

27. Case, D. A.; Darden, T. A.; Cheatham, T. E.; Simmerling, C. L.; Wang, J.; Duke, R. E.; Luo, R.; Walker, R. C.; Zhang, W.; Merz, K. M.; Roberts, B.; Hayik, S.; Roitberg, A.;

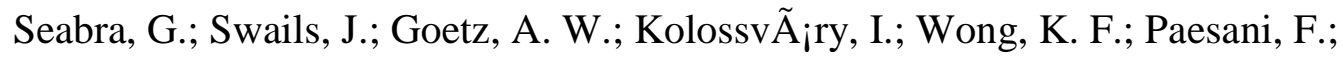
Vanicek, J.; Wolf, R. M.; Liu, J.; Wu, X.; Brozell, S. R.; Steinbrecher, T.; Gohlke, H.; Cai, Q.; Ye, X.; Hsieh, M. J.; Cui, G.; Roe, D. R.; Mathews, D. H.; Seetin, M. G.; Salomon-Ferrer, R.; Sagui, C.; Babin, V.; Luchko, T.; Gusarov, S.; Kovalenko, A.; Kollman, P. A., AMBER 12. In University of California, San Francisco.

28. Kabelac, M.; Kroutil, O.; Predota, M.; Lankas, F.; Sip, M. Phys. Chem. Chem. Phys. 14, 4217-4229.

29. Kirschner, K. N.; Yongye, A. B.; Tschampel, S. M.; González-Outeiriño, J.; Daniels, C. R.; Foley, B. L.; Woods, R. J. J. Comput. Chem. 2008, 29, 622-655.

30. Raju, R. K.; Hillier, I. H.; Burton, N. A.; Vincent, M. A.; Doudou, S.; Bryce, R. A. Physical Chemistry Chemical Physics 2010, 12, 7959-7967.

31. Jorgensen, W. L.; Chandrasekhar, J.; Madura, J. D.; Impey, R. W.; Klein, M. L. J. Chem. Phys. 1983, 79, 926-935.

32. Darden, T.; York, D.; Pedersen, L. J. Chem. Phys. 1993, 98, 10089-10092.

33. Ryckaert, J.-P.; Ciccotti, G.; Berendsen, H. J. C. J. Comput. Phys. 1977, 23, 327-341.

34. Holm, C.; Kremer, K.; Hunenberger, P. Adv. Polym. Sci. 2005, 173, 105-149.

35. Matthews, J. F.; Himmel, M. E.; Brady, J. W. J. Am. Chem. Soc. 2010, 1052, 17-53.

36. Mohan, N.; Vijayalakshmi, K. P.; Koga, N.; Suresh, C. H. J. Comput. Chem. 2010, 31, 2874-2882.

37. Roe, D. R.; Cheatham, T. E. J. Chem. Theory Comput. 2013, 9, 3084-3095.

38. Korth, M.; Pitonak, M.; Rezac, J.; Hobza, P. J. Chem. Theory Comput. 2009, 6, 344-352. 
39. Vincent, M. A.; Hillier, I. H. J. Chem. Inf. Model. 2014, 54, 2255-2260.

40. Gordeev, E. G.; Polynski, M. V.; Ananikov, V. P. Phys. Chem. Chem. Phys. 2013, 15, 18815-18821.

41. Klamt, A.; Schuurmann, G. Chem. Soc., Perkin Trans. 2 1993, 799-805.

42. MOPAC2012, J. J. P. Stewart, Stewart Computational Chemistry, Colorado Springs, CO, USA, OpenMOPAC.net (2012).

43. French, A. D.; Johnson, G. P.; Cramer, C. J.; Csonka, G. I., Conformational analysis of cellobiose by electronic structure theories. Carbohydrate Research 2012, 350, 68-76.

44. Chung, M.-K.; Lee, S. J.; Waters, M. L.; Gagné, M. R. J Am. Chem. Soc. 2012, 134, $11430-11443$

45. Scarpa, F.; Adhikari, S.; Srikantha Phani, A. Nanotechnology 2009, 20, 065709.

46. Ran, J.; Wong, M. W J. Phys. Chem. A 2006, 110, 9702-9709.

47. Matthews, J. F.; Skopec, C. E.; Mason, P. E.; Zuccato, P.; Torget, R. W.; Sugiyama, J.; Himmel, M. E.; Brady, J. W. Carbohydr. Res. 2006, 341, 138-152.

48. Heiner, A. P.; Kuutti, L.; Teleman, O. Carbohydr. Res. 1998, 306, 205-220.

49. Matthews, J. F.; Beckham, G. T.; Bergenstrahle-Wohlert, M.; Brady, J. W.; Himmel, M. E.; Crowley, M. F. J. Chem. Theory Comput. 2012, 8, 735-748.

50. Nishiyama, Y.; Johnson, G. P.; French, A. D.; Forsyth, V. T.; Langan, P. Biomacromolecules 2008, 9, 3133-3140.

51. Newman, R.; Davidson, T. Cellulose 2004, 11, 23-32.

52. Nimlos, M. R.; Beckham, G. T.; Matthews, J. F.; Bu, L.; Himmel, M. E.; Crowley, M. F. J. Biol. Chem. 2012, 287, 20603-20612.

53. Weis, W. I.; Drickamer, K. Annu. Rev. Biochem. 1996, 65, 441-473.

54. Mitchell, F. L.; Miles, S. M.; Neres, J.; Bichenkova, E. V.; Bryce, R. A. Biophys. J. 2010, 98, L38-L40. 
55. Alava, T.; Mann, J. A.; Theodore, C. C.; Benitez, J. J.; Dichtel, W. R.; Parpia, J. M.;

Craighead, H. G. Anal. Chem. 2013, 85, 2754-2759. 
Table 1 All atom RMSD (in $\AA$ ) for each chain in cellulose for different layers in GC100, C100, CG010 and C010; and average RMSD (av) over chains/layers. Corresponding standard deviations in parentheses.

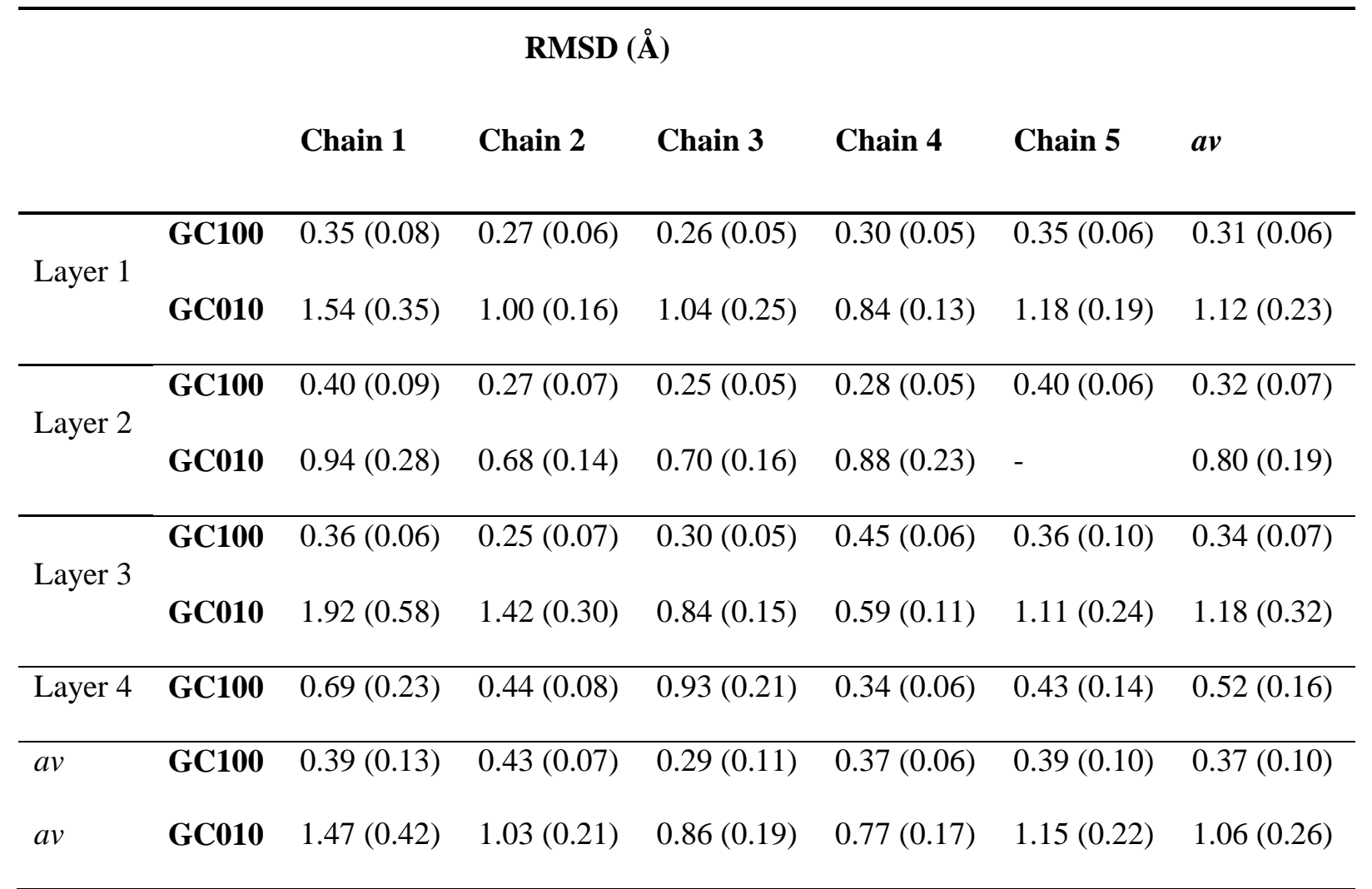


Figure 1. Graphene-cellulose (a) hydrophobic GC100 interfacial model and (b) hydrophilic GC010 model, comprised of layers of decasaccharide chains.

(a)

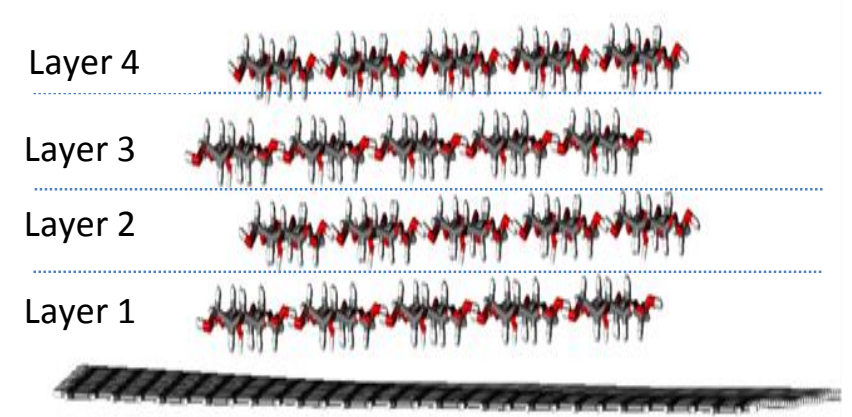

(b)

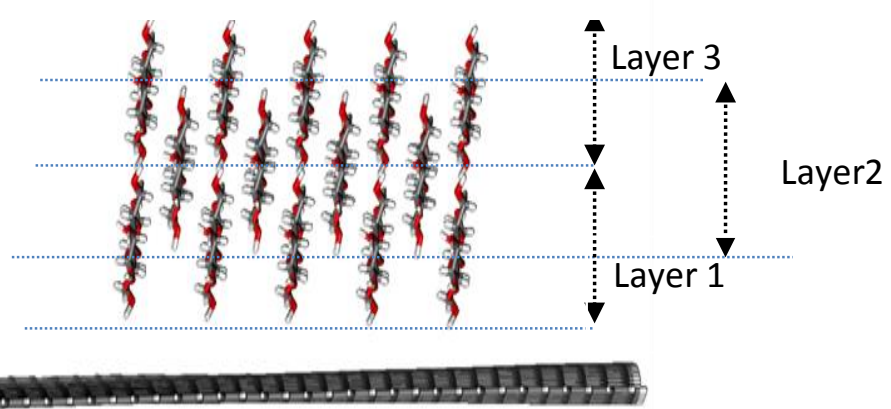


Figure 2. Time series of configurations sampled from 300 ns MD simulation of GC100.

Structures shown of (a) full system (side view), and top views of (b) graphene/layer 1 and (c) graphene/layer 4 .

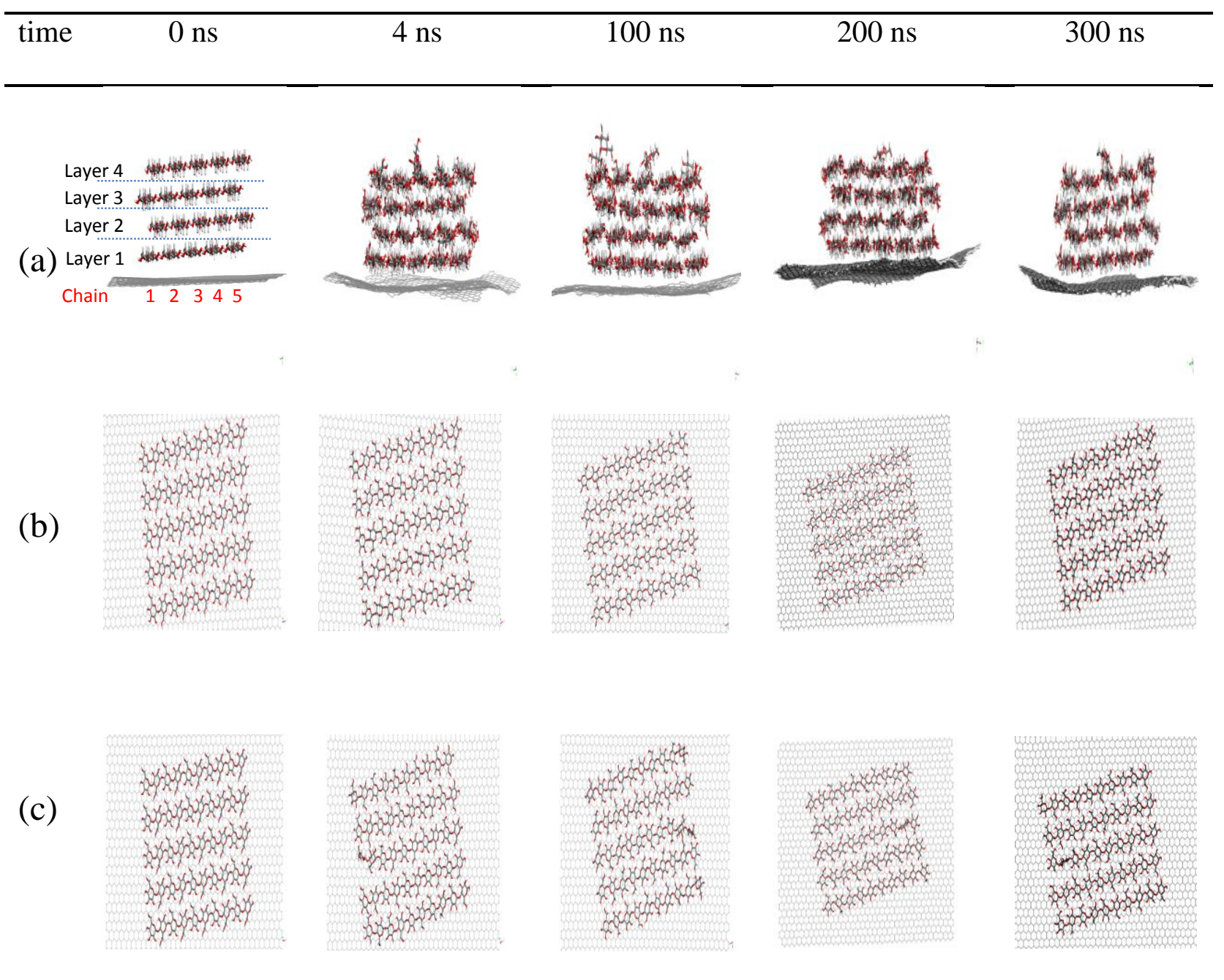


Figure 3. All atom RMSD (in $\AA$ ) for cellulose chains in each layers for (a) GC100 and (b) GC010 backbone during 300 ns MD simulation.

(a)

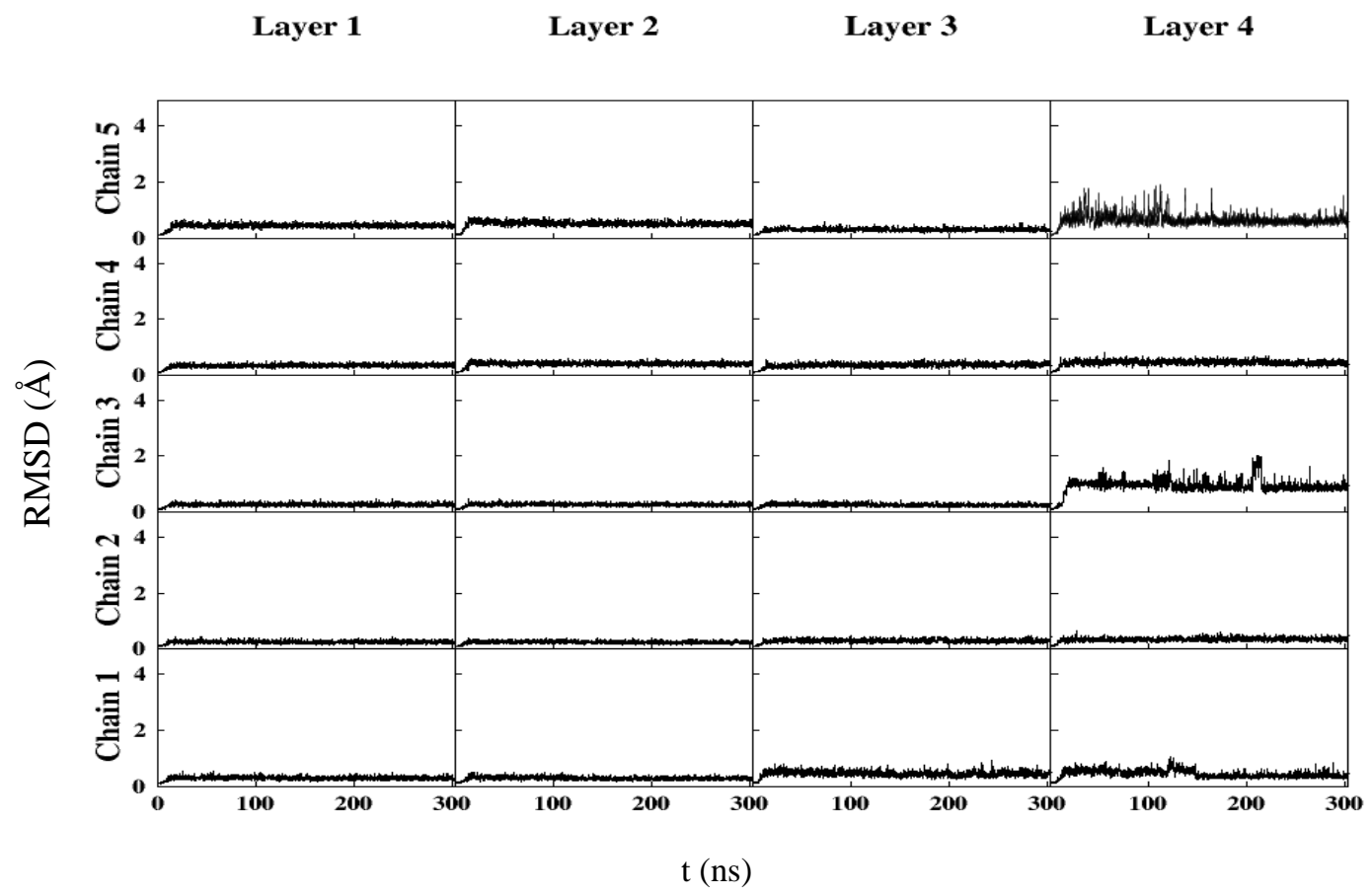

(b)

Layer 1

Layer 2

Layer 3

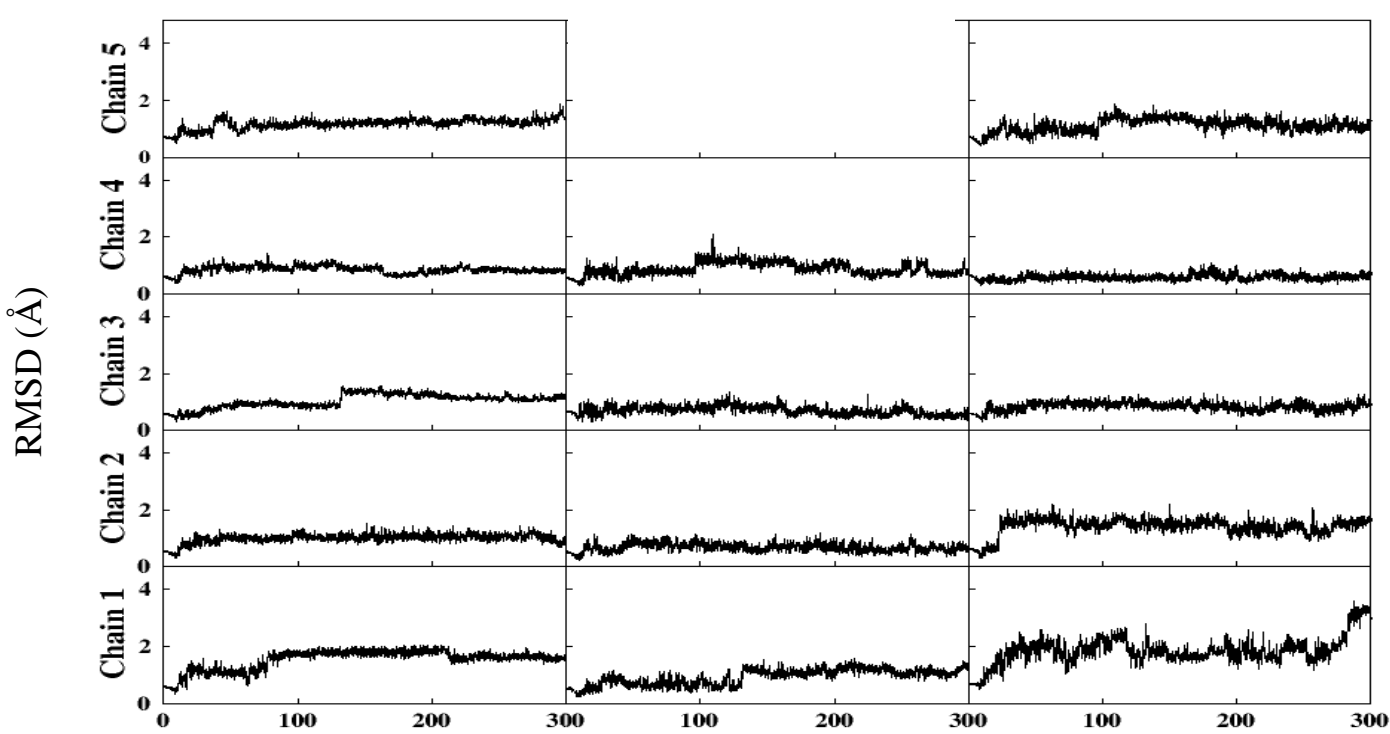

$\mathrm{t}(\mathrm{ns})$ 
Figure 4. Number of cellulose-graphene $\mathrm{CH}-\pi$ and $\mathrm{OH}-\pi$ interactions $(n)$ for GC100 and GC010 during 300 ns MD simulation.

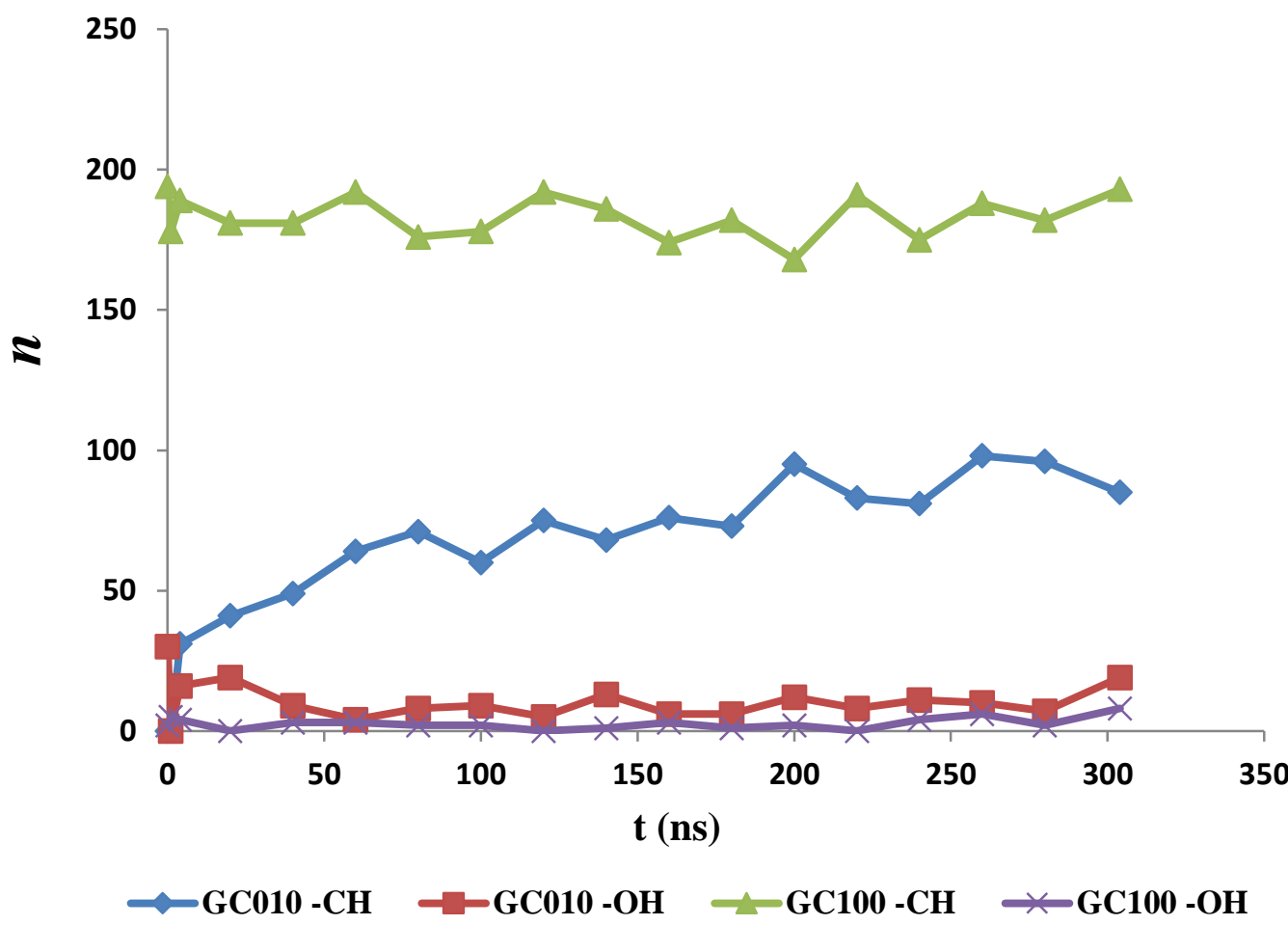


Figure 5. Cellulose-graphene adhesion energy $E_{\text {inter }}\left(\right.$ in $\mathrm{kcal} \mathrm{mol}^{-1}$ ) over $300 \mathrm{~ns}$ trajectory for GC100 and GC010 models in (a) vacuum and (b) implicit solvent.

(a)

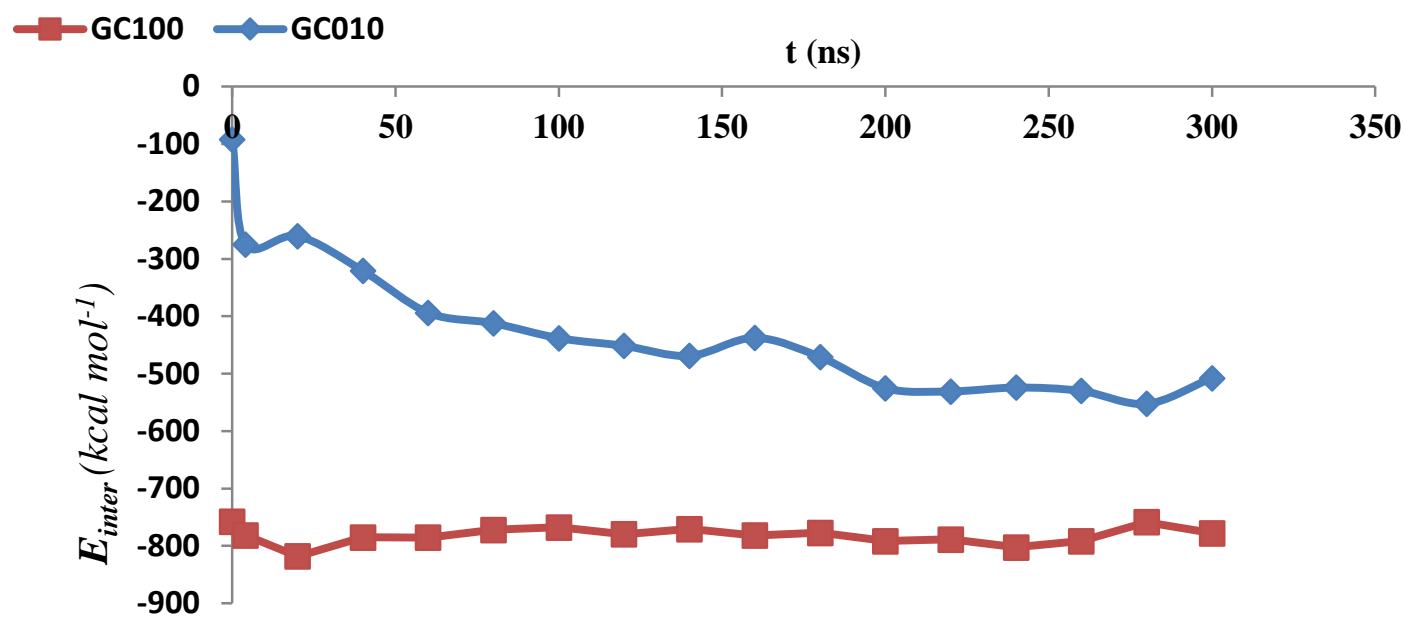

(b)

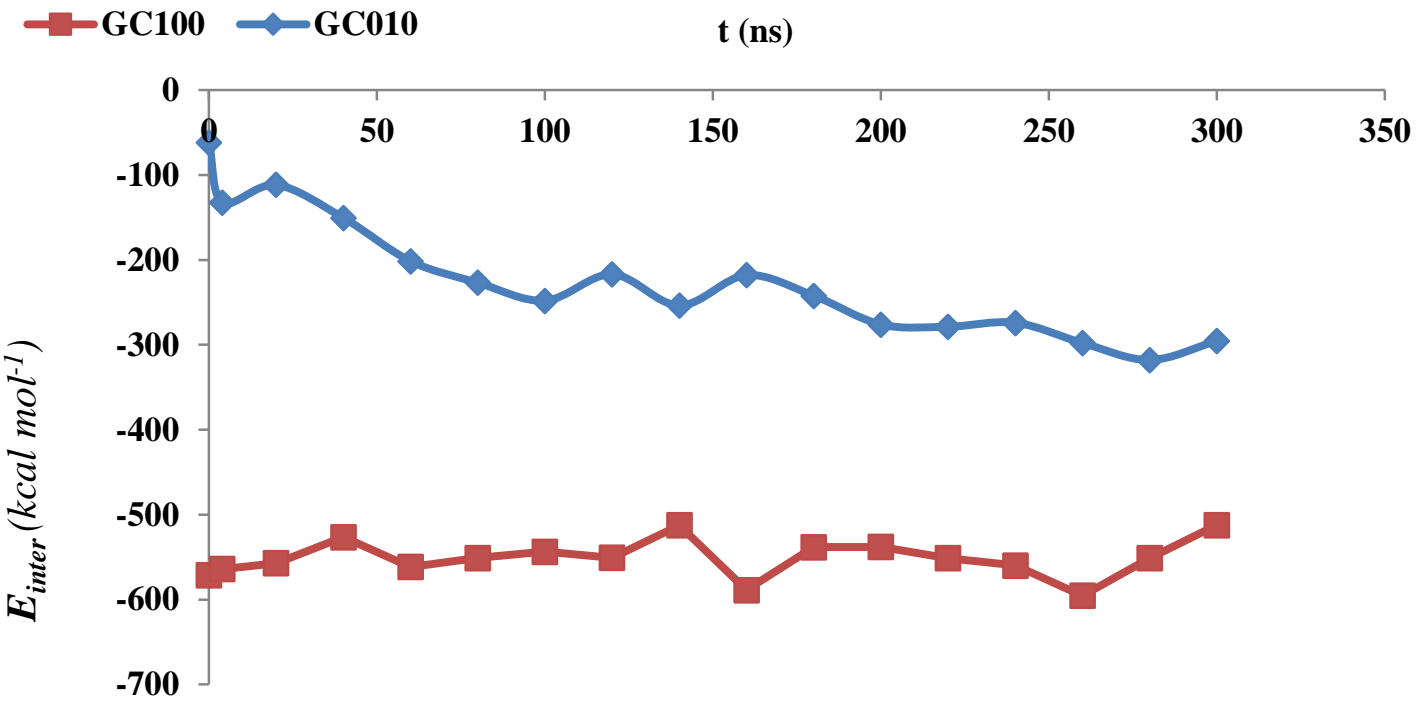


Figure 6. Water number density in GC100 and GC010 over 300 ns MD simulation: (a) average, (b) $0 \mathrm{~ns}$, (c) $50 \mathrm{ns,} \mathrm{(d)} 100 \mathrm{ns,} \mathrm{(e)} 100 \mathrm{~ns}$ side views, (f) $200 \mathrm{~ns}$ and (g) $300 \mathrm{~ns}$. Cellulose and graphene omitted in some images for clarity.

(a)
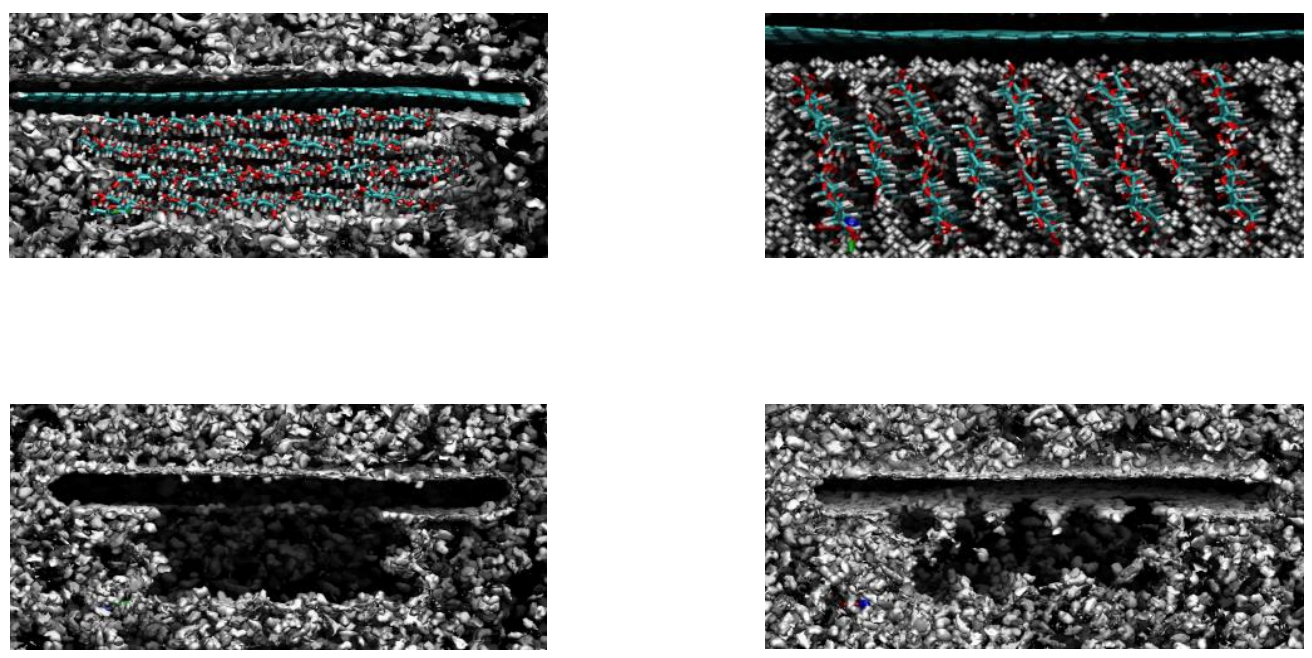

(b)

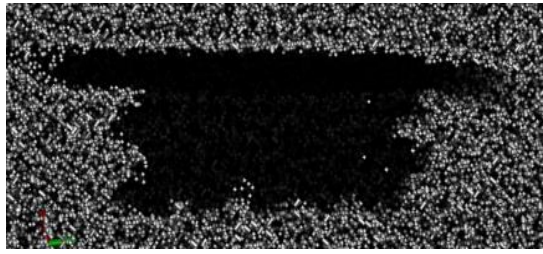

(c)
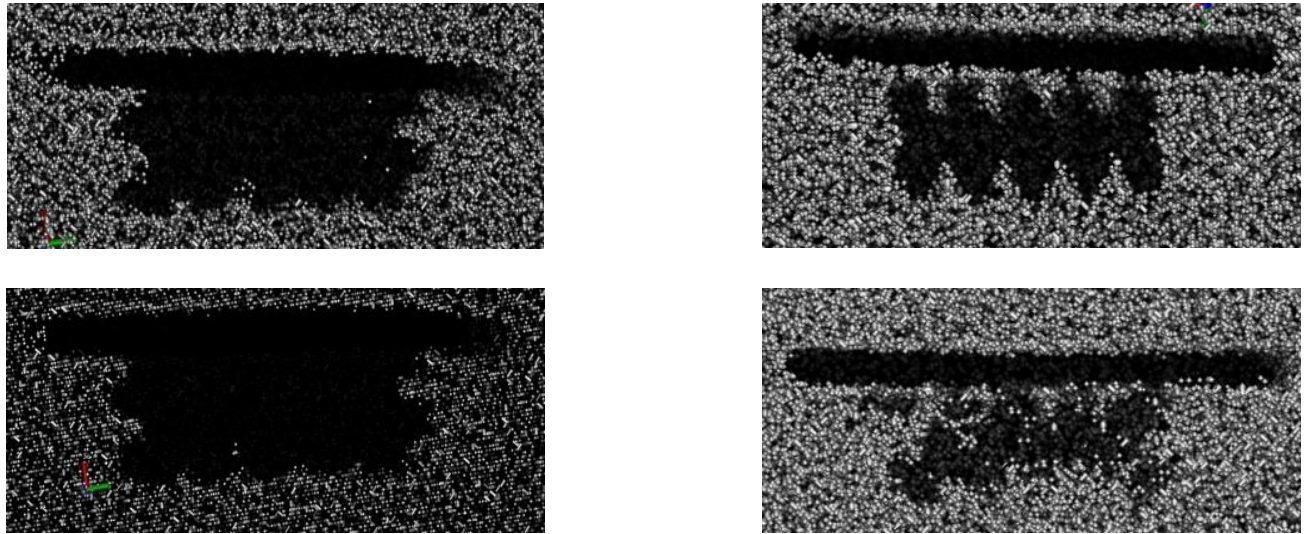

(d)

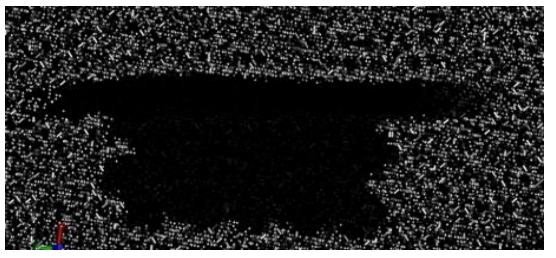

(e)
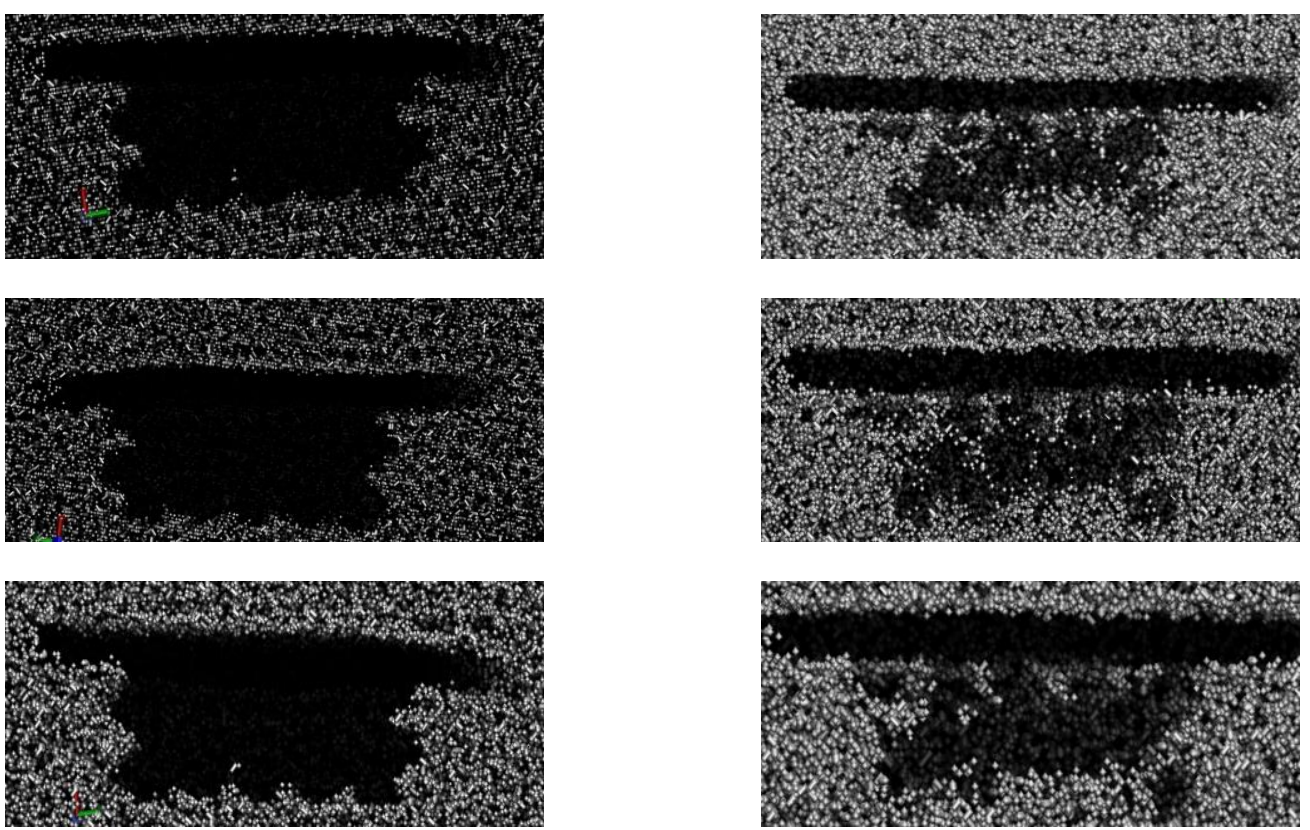
(f)
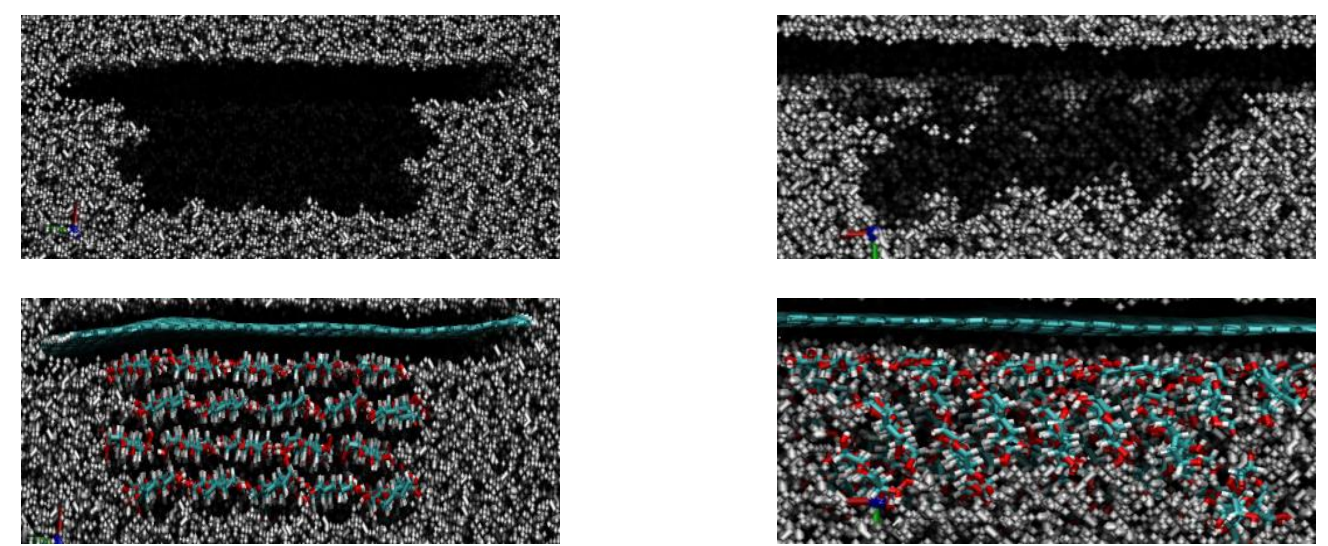
Figure 7. Glycosidic angles $\varphi$ (left) and $\psi$ (right) for (a) GC100 and (b) GC010 during 300 ns MD simulation for chain 5 in layer 1 . Angles are in degrees.

(a)

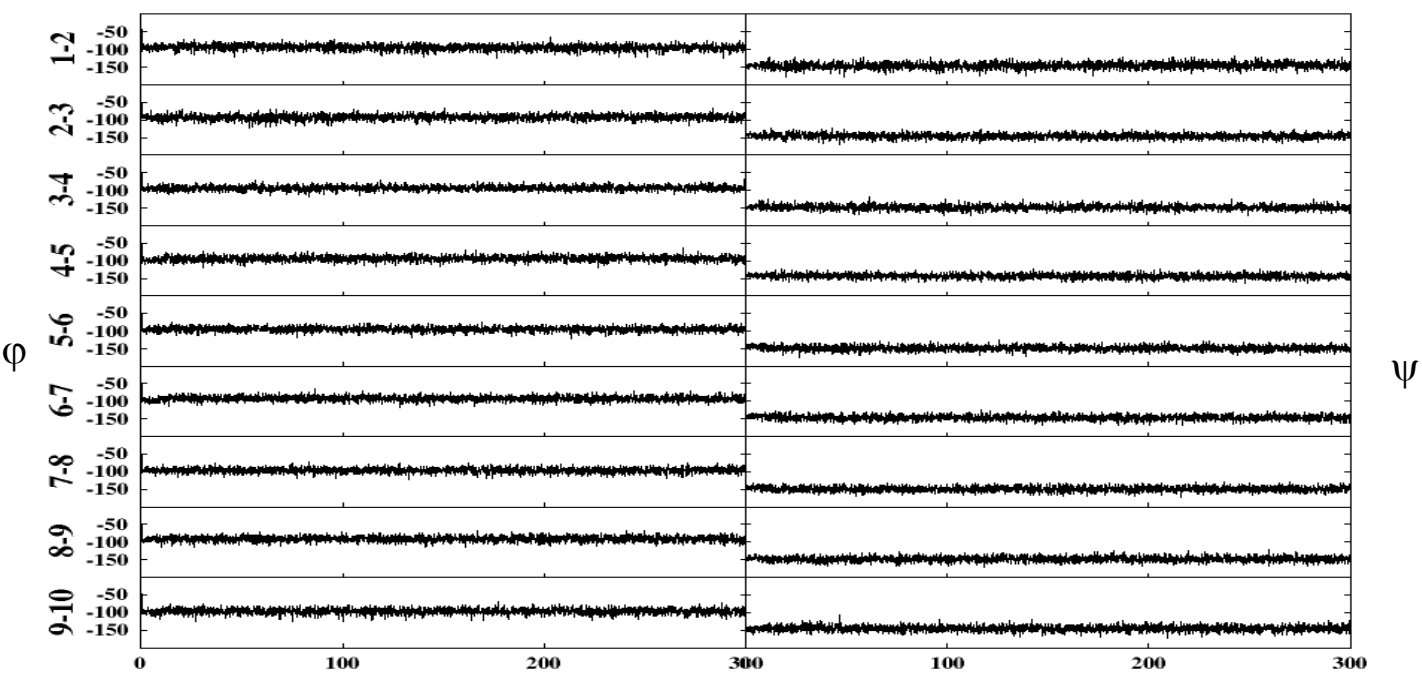

$\mathrm{t}(\mathrm{ns})$

(b)

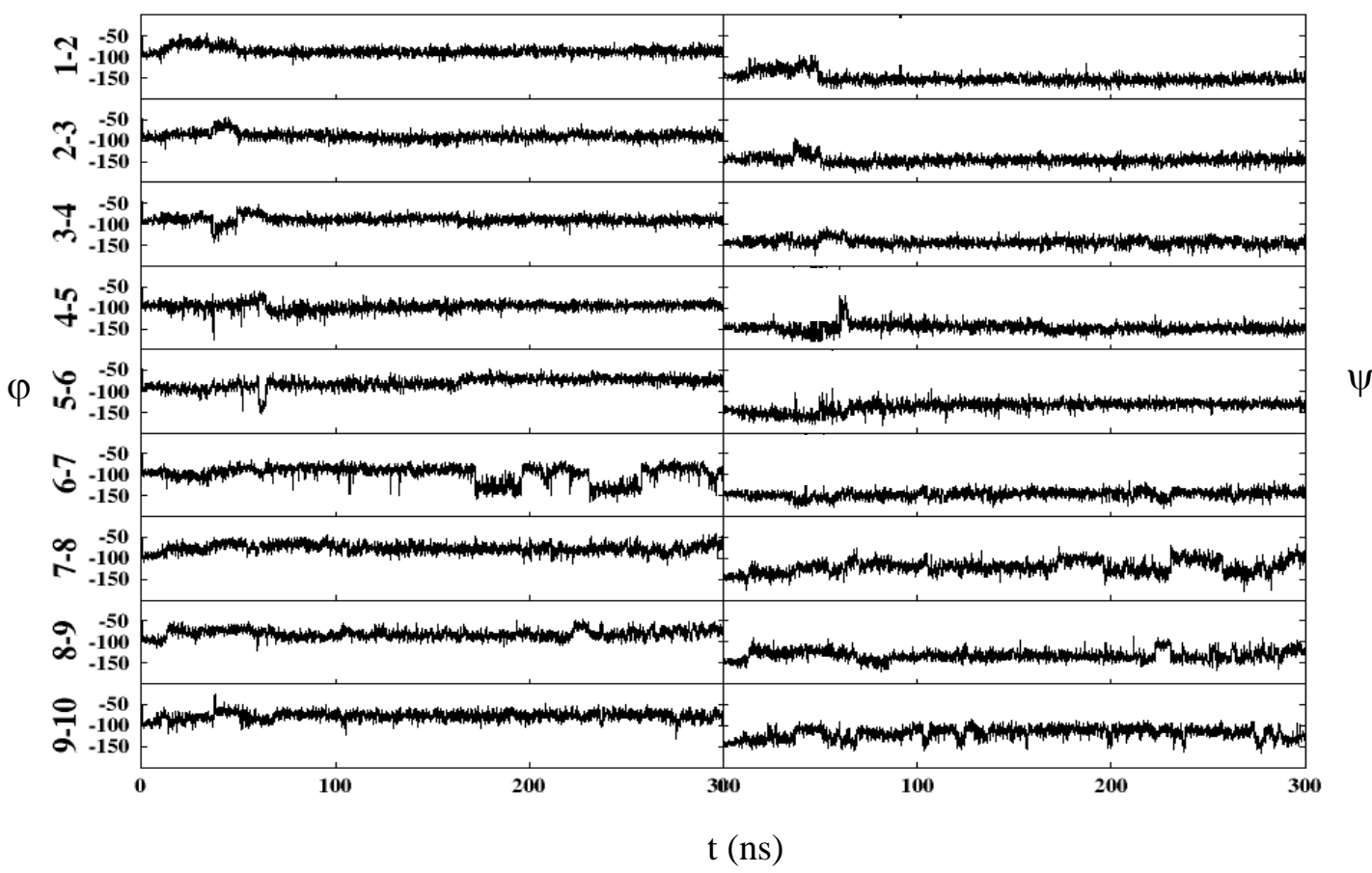


Figure 8. Predominant orientation of C6 hydroxymethyl group in chain for (a) GC100 and (b) GC010 during 300 ns MD simulation.

(a)

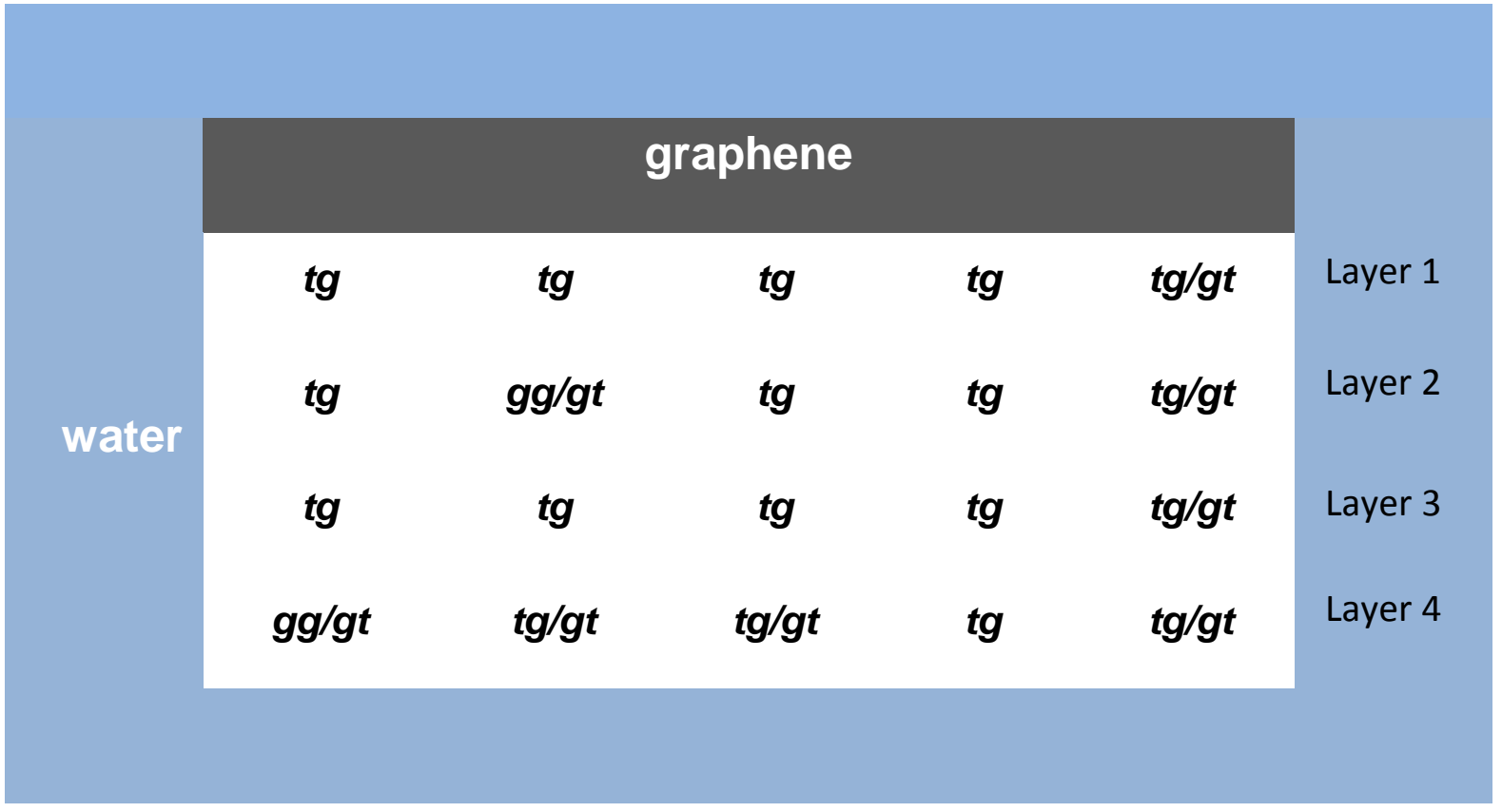

(b)

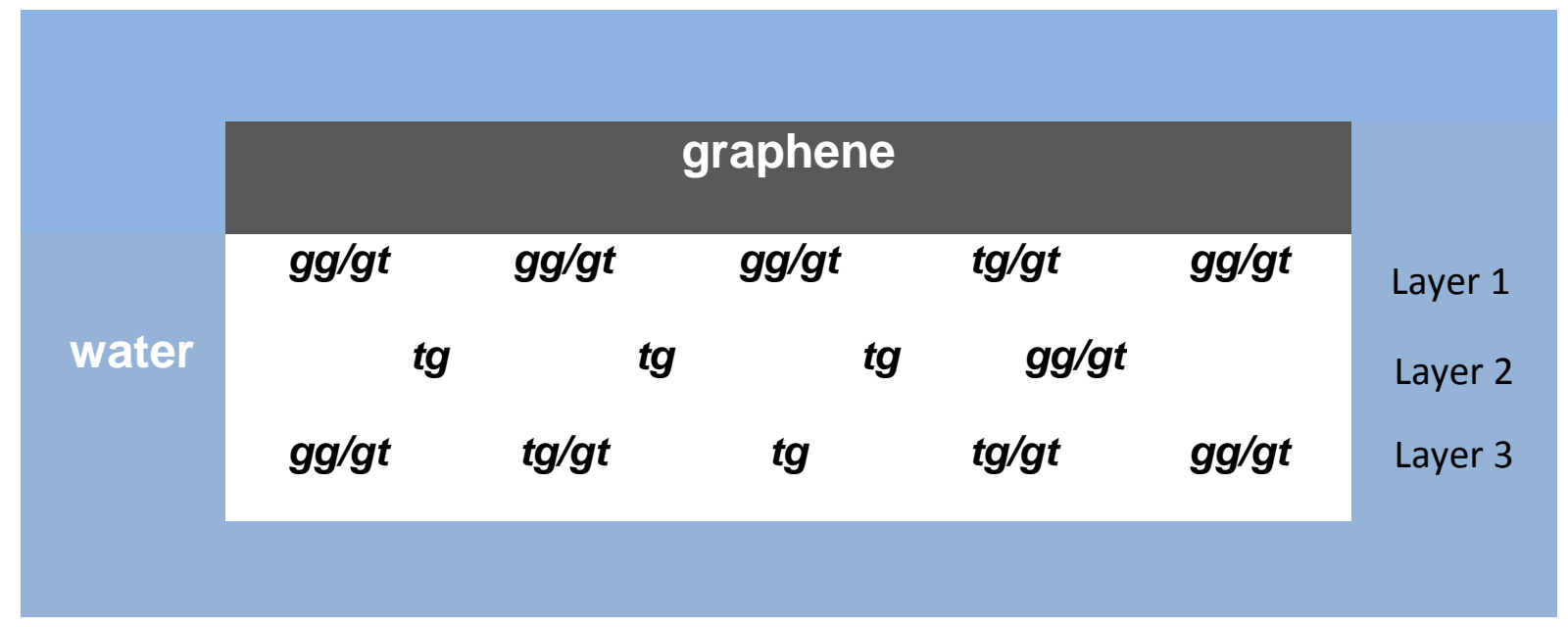


Figure 9. The number of intramolecular $\left(n_{\text {intra }}\right)$ and intermolecular hydrogen bonds per decasaccharide chain $\left(n_{\text {inter }}\right)$ in layer 1 and layer 4 , as defined according to the initial crystallographic hydrogen bond network, for (a) GC100 and layer 1 and layer 3 for (b) GC010.

(a) GC100

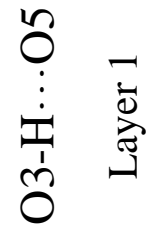

$\underset{5}{\stackrel{5}{5}}$
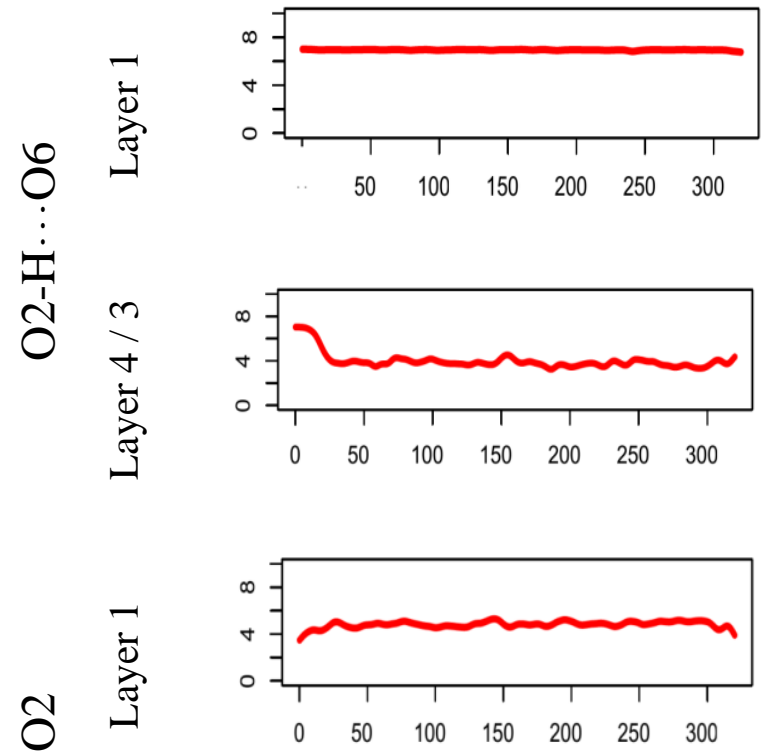

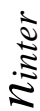

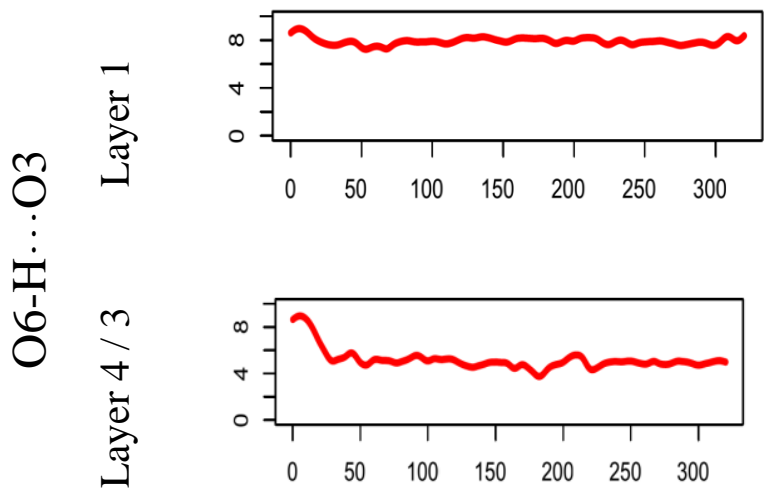

(b) GC010
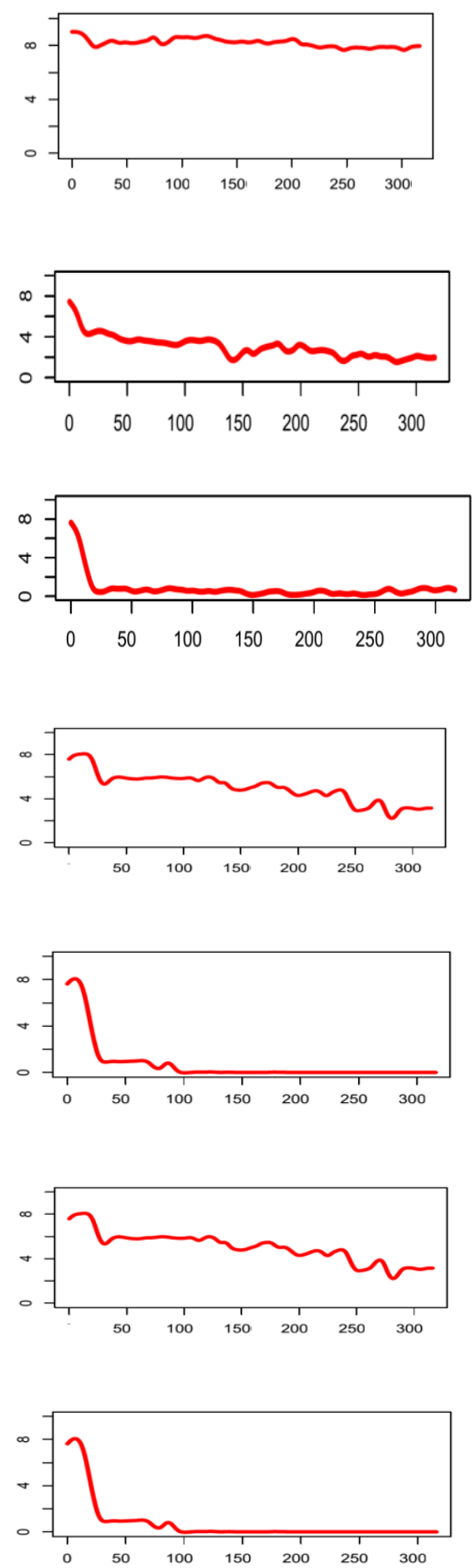
Figure 10. Time series of snapshots of GC010 over 300 ns simulation, for total solute in side view (a,p) and top view of layer 1 on graphene (b-o). Reoriented glucosyl units (yellow) and descended chain (orange) also indicated. Chain numbering indicated in (b).

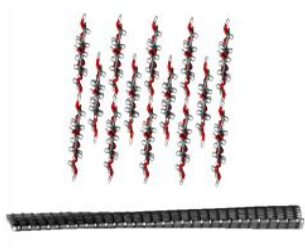

a) $0 \mathrm{~ns}($ side $)$

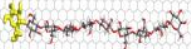

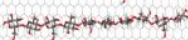

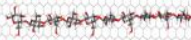

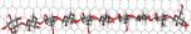

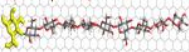

e) $5 \mathrm{~ns}$

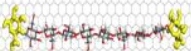

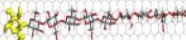

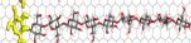

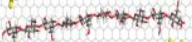

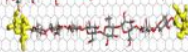

i) $\quad 19.9 \mathrm{~ns}$

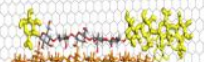

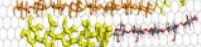

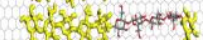

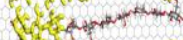

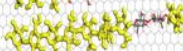
m) $104 \mathrm{~ns}$

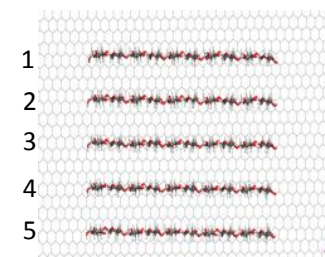

b) $0 \mathrm{~ns}$ (top)

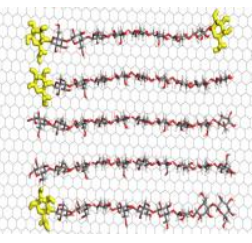

f) $11 \mathrm{~ns}$

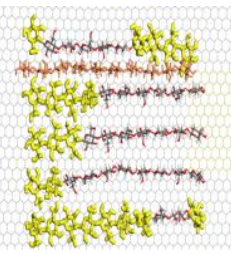

j) $20 \mathrm{~ns}$

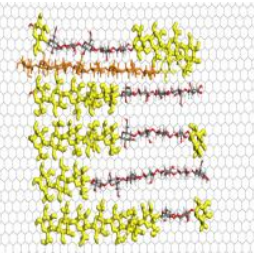

n) $184 \mathrm{~ns}$

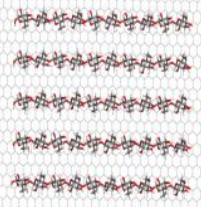

c) $3 \mathrm{~ns}$

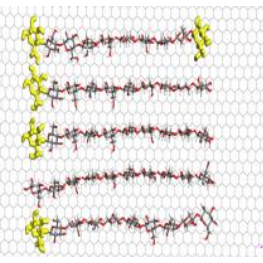

g) $14 \mathrm{~ns}$

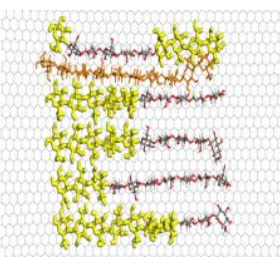

k) $54 \mathrm{~ns}$

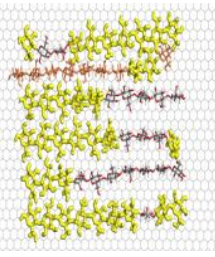

o) $304 \mathrm{~ns}$

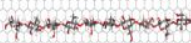

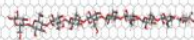

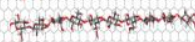

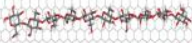

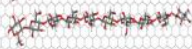

d) $4 \mathrm{~ns}$

\section{3.

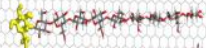

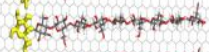 thet

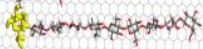

h) $19 \mathrm{~ns}$

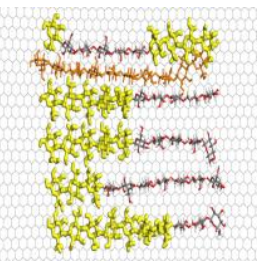

1) $74 \mathrm{~ns}$

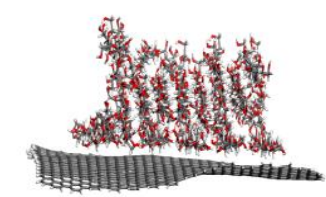

p) $304 \mathrm{~ns}$ 
Figure 11. Snapshot geometry of GC010 at 100 ns from 300 ns MD simulation. Glucosyl residues of cellulose in green and graphene rings in orange.

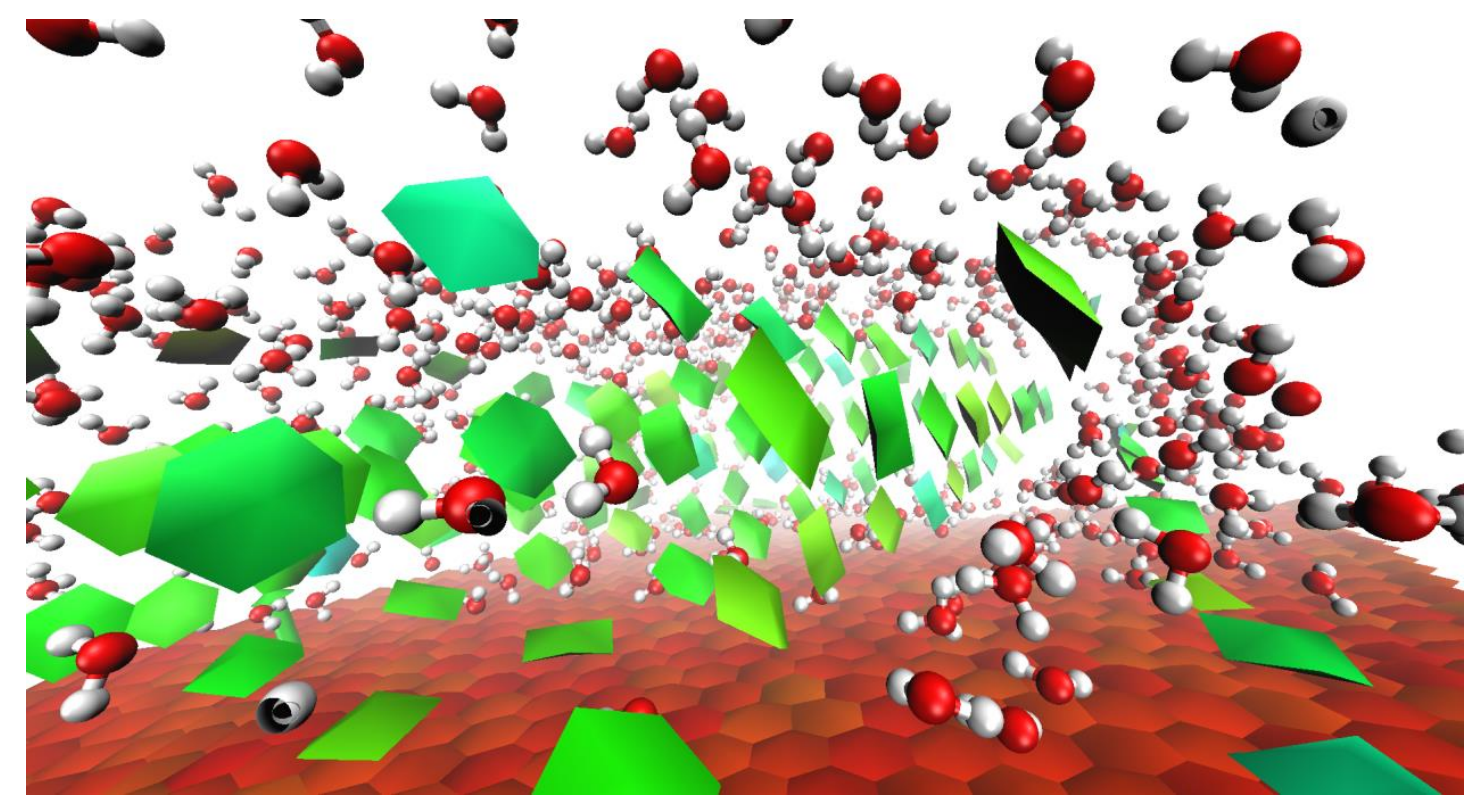


Figure 12. Number of newly formed interchain hydrogen bonds in GC010 $\left(n_{\text {inter }}\right)$ between chains in layer in $300 \mathrm{~ns}$ MD simulation.

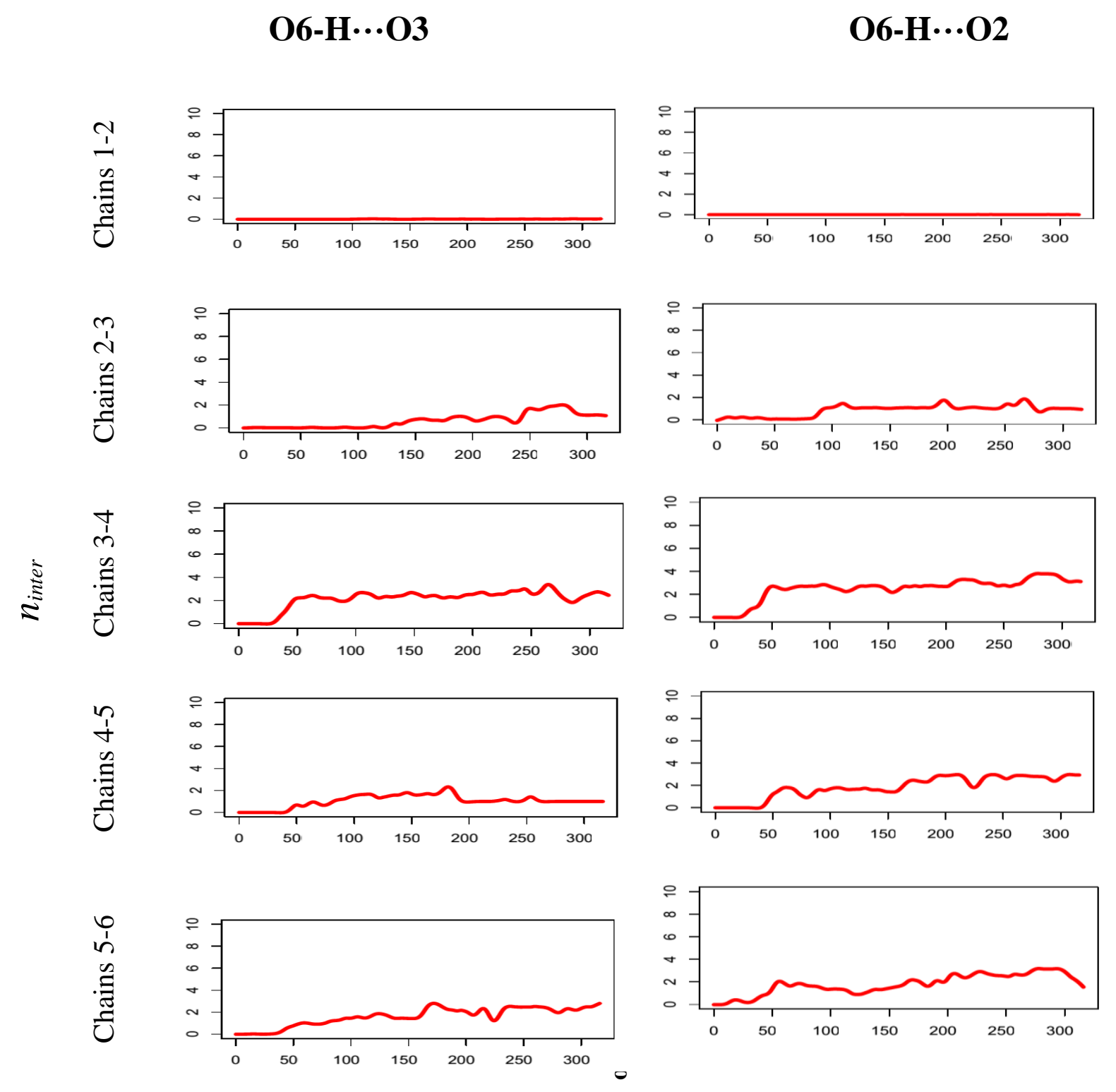


Figure 9. Snapshots from (a) $300 \mathrm{~ns}$ and (b) $100 \mathrm{~ns}$ MD simulation of GC010 that shows breakage of interchain hydrogen bonds between chain 1 in layer 1 and chain 1 in layer 3 .

(a)

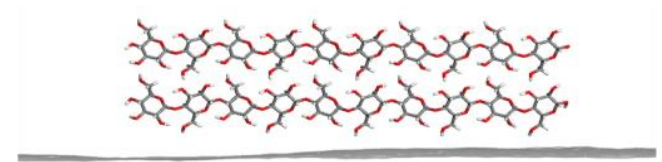

$0 \mathrm{~ns}$

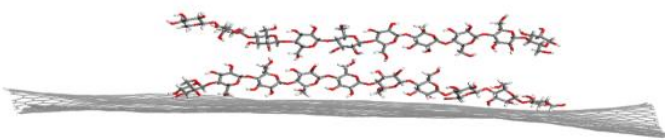

14 ns

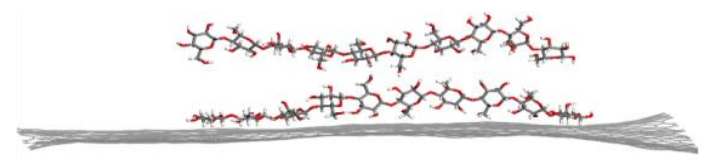

34 ns

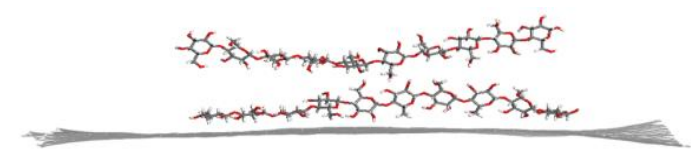

$104 \mathrm{~ns}$

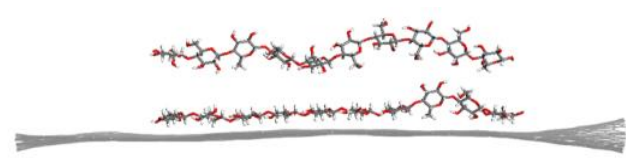

$200 \mathrm{~ns}$

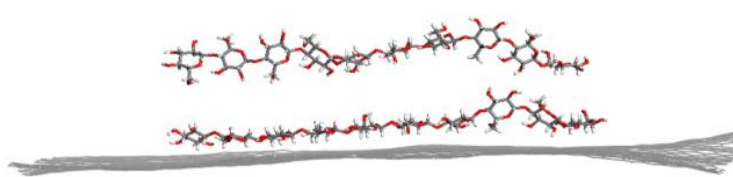

$224 \mathrm{~ns}$

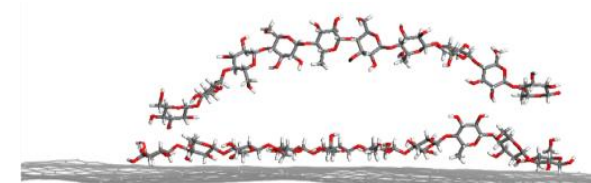

$304 \mathrm{~ns}$ (b)

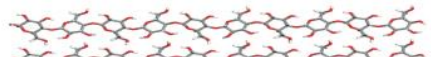

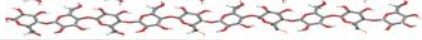

$0 \mathrm{~ns}$
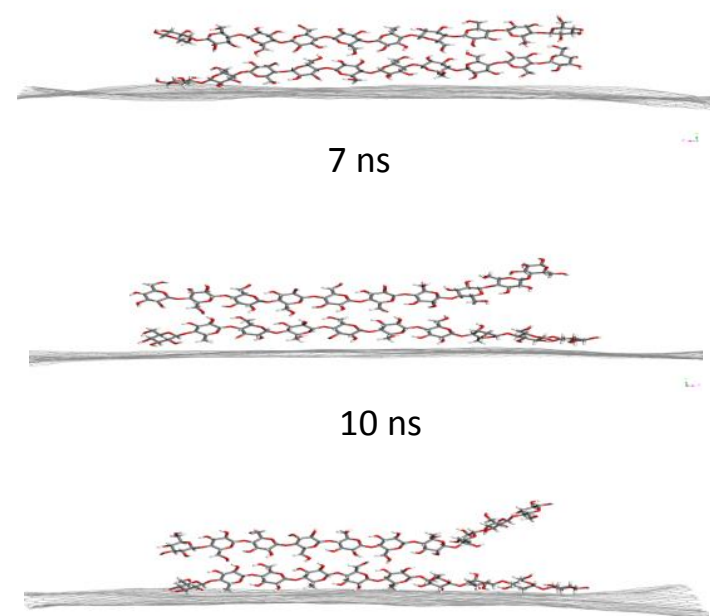

15 ns

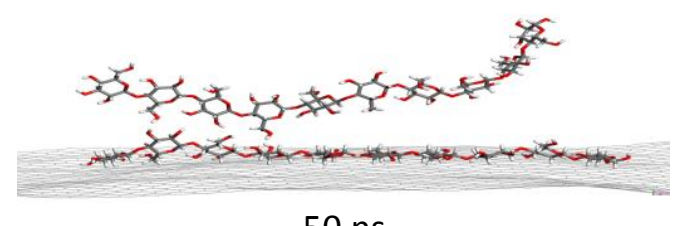

$50 \mathrm{~ns}$

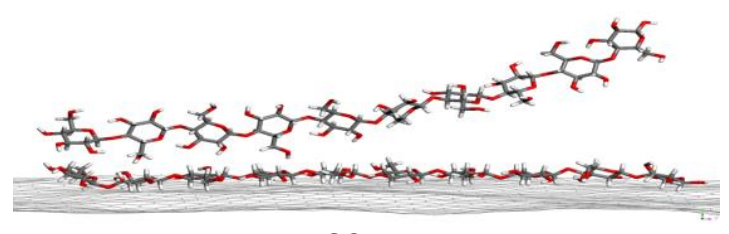

80 ns

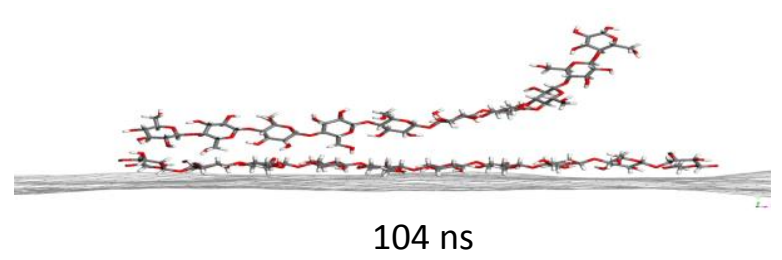



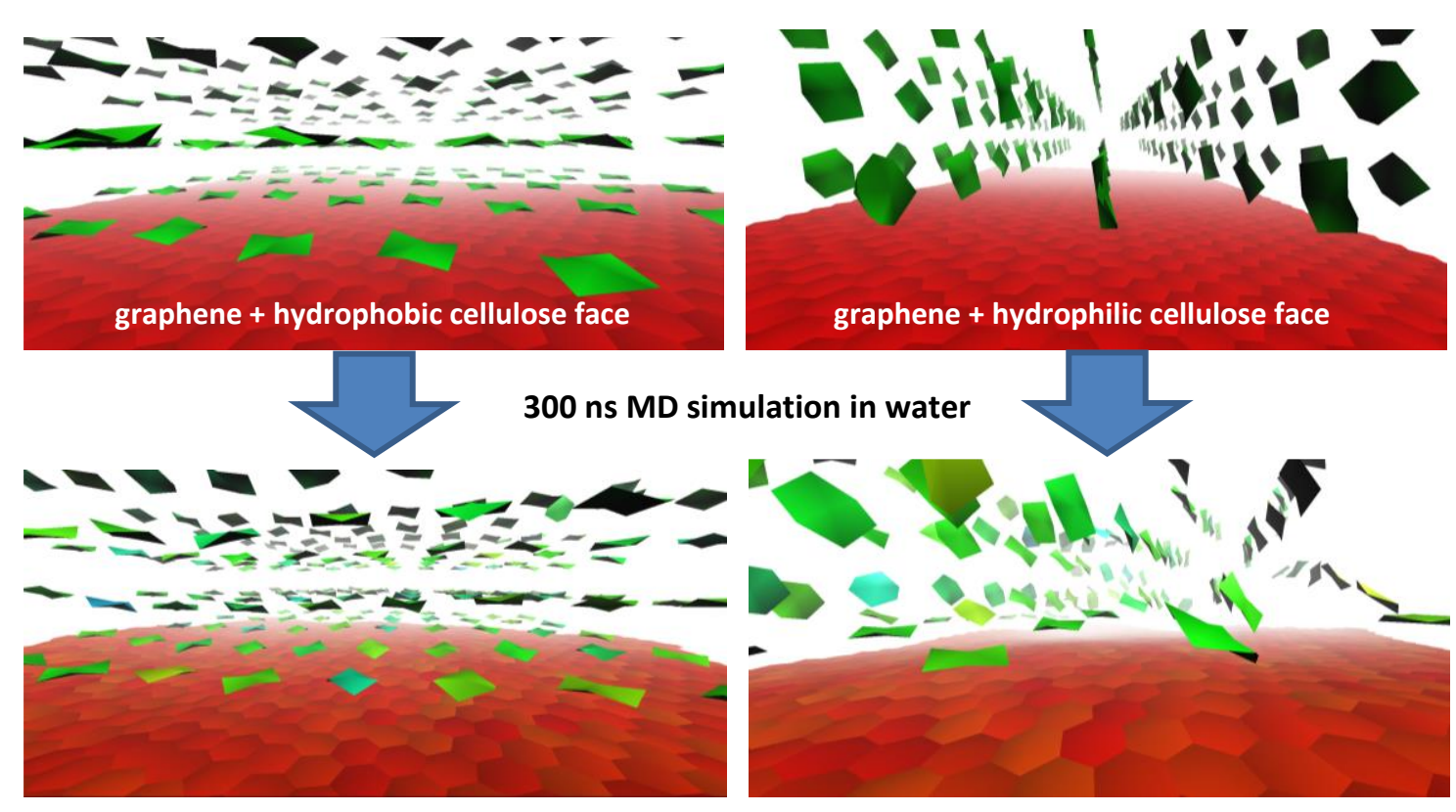

graphene + hydrophilic cellulose face
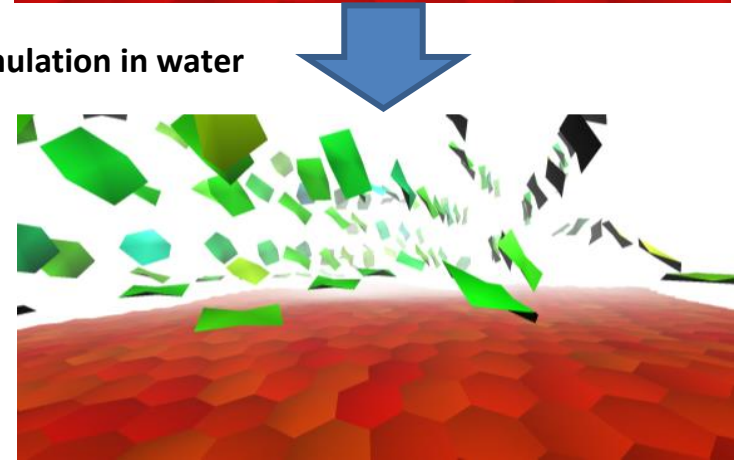